\title{
Growth of Cloud Droplets by Turbulent Collision-Coalescence
}

\author{
Yan Xue AND Lian-Ping Wang \\ Department of Mechanical Engineering, University of Delaware, Newark, Delaware \\ WOJCIECH W. GrabOWSKI \\ Mesoscale and Microscale Meteorology Division, National Center for Atmospheric Research,* Boulder, Colorado
}

(Manuscript received 19 January 2007, in final form 22 May 2007)

\begin{abstract}
An open question in cloud physics is how rain forms in warm cumulus as rapidly as it is sometimes observed. In particular, the growth of cloud droplets across the size gap from 10 to $50 \mu \mathrm{m}$ in radius has not been fully explained. In this paper, the authors investigate the growth of cloud droplets by collisioncoalescence, taking into account both the gravitational mechanism and several enhancements of the collision-coalescence rate due to air turbulence. The kinetic collection equation (KCE) is solved with an accurate bin integral method and a newly developed parameterization of turbulent collection kernel derived from direct numerical simulation of droplet-laden turbulent flows. Three other formulations of the turbulent collection kernel are also considered so as to assess the dependence of the rain initiation time on the nature of the collection kernel. The results are compared to the base case using the Hall hydrodynamicalgravitational collection kernel. Under liquid water content and eddy dissipation rate values typical of small cumulus clouds, it is found that air turbulence has a significant impact on the collection kernel and thus on the time required to form drizzle drops. With the most realistic turbulent kernel, the air turbulence can shorten the time for the formation of drizzle drops by about $40 \%$ relative to the base case, applying measures based on either the radar reflectivity or the mass-weighted drop size. A methodology is also developed to unambiguously identify the three phases of droplet growth, namely, the autoconversion phase, the accretion phase, and the larger hydrometeor self-collection phase. The important observation is that even a moderate enhancement of collection kernel by turbulence can have a significant impact on the autoconversion phase of the growth.
\end{abstract}

\section{Introduction}

Cloud droplets of radii less than 10-15 $\mu \mathrm{m}$ grow efficiently through diffusion of water vapor, and droplets larger than $30-50 \mu \mathrm{m}$ in radii grow efficiently through gravitational collisions (Langmuir 1948; Kogan 1993; Beard and Ochs 1993; Pruppacher and Klett 1997). An open question is why rain forms in warm (i.e., ice free) cumulus clouds as rapidly as it has sometimes been observed. Observations of radar reflectivity in tropical re-

\footnotetext{
* The National Center for Atmospheric Research is sponsored by the National Science Foundation.
}

Corresponding author address: Lian-Ping Wang, Department of Mechanical Engineering, 126 Spencer Laboratory, University of Delaware, Newark, DE 19716-3140.

E-mail: lwang@udel.edu

DOI: $10.1175 / 2007 J A S 2406.1$

(C) 2008 American Meteorological Society gions suggest that rain could form in cumulus clouds by the warm rain process in approximately 15-20 min (Szumowski et al. 1997; Knight et al. 2002). While there are still uncertainties in linking radar reflectivity and the rain initiation time as the radar reflectivity depends on both the average droplet size and the liquid water content (Knight et al. 2002), the above rain initiation time is usually quoted as the average time for the initiation of warm rainfall in unpolluted, maritime clouds (i.e., from the initial development of a cumulus cloud to the first appearance of rain). Theoretical predictions based on the gravitational-coalescence mechanism alone would require a time interval on the order of an hour for droplets to grow from 20 to $100 \mu \mathrm{m}$ in radius (the actual time depends on the cloud water content, initial droplet size spectrum, updraft speed, etc.; see Pruppacher and Klett 1997). Therefore, there appears to be a factor of 2 or more difference between the predicted growth time and the observed growth time. 
In general, it is difficult to explain the rapid growth of cloud droplets in the size range from 10 to $50 \mu \mathrm{m}$ in radius (i.e., the so-called size gap) for which neither the condensation nor the gravitational collision-coalescence mechanism is effective. The onset of drizzle-size $(\sim 100 \mu \mathrm{m}$ in radius) raindrops is still poorly understood in many precipitating cloud systems.

Several mechanisms have been considered in the past to explain the rapid development of rain in shallow convective clouds (Beard and Ochs 1993; Pruppacher and Klett 1997; Khain et al. 2000). The first mechanism involves entrainment of dry environmental air into the cloud. Although entrainment lowers the cloud water content (and thus has a negative impact on rain development), it can result in a dramatic impact on cloud droplet spectra. In particular, broad spectra are typically produced as a result of entrainment and mixing (Brenguier and Grabowski 1993; Su et al. 1998; LasherTrapp et al. 2005), but the resulting effects on large droplet formation and coalescence initiation remain unknown. The second mechanism involves effects of giant aerosol particles that allow quicker formation of large cloud droplets, thereby initiating coalescence sooner (Johnson 1982; Blyth et al. 2003). Srivastava (1989) suggested that the droplet spectral width can be broadened by considering local values of the water vapor supersaturation rather than the mean supersaturation that a large population of cloud droplets experience. However, numerical results by Vaillancourt et al. (2001, 2002) suggest that this effect contributes insignificantly to the width of the cloud droplet spectrum. The fourth mechanism concerns effects of air turbulence on the relative motion of droplets, concentration fluctuations, and collision efficiencies (Khain et al. 2000; Franklin et al. 2005; Wang et al. 2005a). Compounding the problem is that the commonly used kinetic collection equation for modeling the time evolution of droplet size distribution is not fully consistent with the stochastic nature of the collision-coalescence process (Telford 1955; Scott 1967; Gillespie 1972, 1975; Kostinski and Shaw 2005; Wang et al. 2006b).

This paper concerns the effects of air turbulence on the growth of cloud droplets by collision-coalescence, a key step in the formation of warm rain (Pruppacher and Klett 1997). In general, the rate of collisions is controlled by the gravitational mechanism for rain drops of radius larger than $50 \mu \mathrm{m}$, but air turbulence could significantly enhance the collision rate for cloud droplets (from 10 to $30 \mu \mathrm{m}$ in radius) and the intermediate size range between small cloud droplets to rain drops (from 30 to $100 \mu \mathrm{m}$ in radius).

Historically, there were several attempts to analyze the effects of air turbulence on the collision rates of cloud droplets. Arenberg (1939) is perhaps the first to recognize qualitatively that air turbulence can increase the relative motion of cloud droplets, followed by semianalytical studies of Gabilly (1949) and East and Marshall (1954). Saffman and Turner (1956) developed a rigorous theoretical formulation for the enhanced relative motion by turbulence on collision rates, applicable to weak-inertia droplets. Reuter et al. (1988) introduced a stochastic model and showed that turbulent fluctuations could enhance the relative motion and the geometric collision kernel. Another aspect that had been studied in the context of cloud droplets concerns the effect of turbulent motion on the collision efficiency of cloud droplets (de Almeida 1976, 1979; Grover and Pruppacher 1985; Koziol and Leighton 1996). These earlier studies are somewhat limited in terms of their quantitative capabilities either because of the assumptions made to simplify the equation of motion of droplets or the inaccurate methods employed to describe the turbulent fluctuations. For example, de Almeida (1979) suggested that turbulence could increase the collision efficiency of cloud droplets less than $40 \mu \mathrm{m}$ in radius by a factor as much as 26 relative to the still-air case, but he inappropriately used the inertial-range rather than dissipation-range scaling to quantify the relative motion caused by air turbulence (Grover and Pruppacher 1985).

Over the last 10 years, an increasing number of studies have been reported in both engineering and atmospheric literature concerning the collision rate of particles in a turbulent flow. These studies suggest that the collection kernel of cloud droplets can be enhanced by several effects of turbulence, including 1) enhanced relative motion due to differential acceleration and shear effects (Kruis and Kusters 1997; Pinsky and Khain 1997; Sundaram and Collins 1997; Wang et al. 2000; Zhou et al. 2001, hereafter ZWW01; Dodin and Elperin 2002), 2) enhanced average pair density due to local preferential concentration of droplets (Sundaram and Collins 1997; Wang et al. 2000; ZWW01; Zaichik and Alipchenkov 2003; Zaichik et al. 2003), 3) enhancement due to selective alterations of the settling rate by turbulence (Wang and Maxey 1993; Dávila and Hunt 2001; Ghosh and Jonas 2001), and 4) enhanced collision efficiency (Pinsky et al. 1999, 2000; Wang et al. 2005a). The levels of enhancement depend, in a complex manner, on the size of droplets (which in turn determines the response time and settling velocity) and the strength of air turbulence (i.e., the dissipation rate, Reynolds number, etc.).

While all the studies consistently point to collision enhancements by air turbulence, the parameter space 
considered typically does not match the conditions of cloud droplets [see discussions in Grabowski and Vaillancourt (1999) and Vaillancourt and Yau (2000)], and many of these studies do not consider the gravity force acting on the droplets. Combined with different approximations employed, these issues lead to uncertain estimates of the exact level of collision-rate enhancement by air turbulence. This is related to the complexity of collisional interactions in a turbulent flow and the lack (or inaccuracy) of direct measurements of turbulence and droplet distribution in clouds. Recent observations and analyses of observational data from the Fast Forward Scattering Spectrometer probe (FSSP; Brenguier et al. 1998) suggest some evidence that cloud droplets inside adiabatic cores may not be distributed randomly in space, but the conclusions have not been consistent and the issue remains largely unresolved (Chaumat and Brenguier 1998; Grabowski and Vaillancourt 1999; Jameson and Kostinski 2000; Kostinski and Shaw 2001; Pinsky and Khain 2002a).

Recently, systematic studies of the collision kernel for cloud droplets have been undertaken either through direct numerical simulations (Franklin et al. 2005; Wang et al. 2005a, 2006c; Ayala 2005, hereafter A05) or a kinematic representation of turbulence (Pinsky et al. 1999; Pinsky and Khain 2004; Pinsky et al. 2006). These studies provide not only quantitative data on turbulent collision kernels but also a better understanding of the interaction of cloud droplets with air turbulence. They could eventually lead to a general and accurate parameterization of turbulent collision kernels including the effects of air turbulence on both geometric collision rate and collision efficiency. In a recent study, A05 developed an analytical model for the geometric collision rate of cloud droplets based on direct numerical simulations of collisions of sedimenting droplets in the context of cloud turbulence. This is a step forward when compared to the previous models of ZWW01 and Wang et al. (2000) who considered only nonsettling particles.

Several groups have also attempted to address the impact of selected aspects of air turbulence on the time evolution of the droplet size spectrum. Pinsky and Khain (1997) and Pinsky and Khain (2002b) showed that collision kernels taking into account the effect of air turbulence on the relative motion of droplets could lead to the acceleration of large droplets and raindrop formation. Falkovich et al. (2002) argued that preferential concentration of droplets and local fluid acceleration of cloud turbulence could substantially accelerate the appearance of large droplets that trigger rain. Ghosh et al. (2005) demonstrated that the enhanced sedimentation velocity due to air turbulence could grow droplets rapidly from 20 to $80 \mu \mathrm{m}$ and that this mecha- nism does not depend on the level of cloud turbulence. The above studies, however, do not include all known aspects of air turbulence on the geometric collision rate in their investigation of droplet size spectrum. Moreover, the turbulent collision kernel used in these studies is derived from either qualitative or empirical formulations of air turbulence.

The objective of this paper is to advance current understanding of how the effects of air turbulence on the geometric collision kernel alter the size evolution of cloud droplets, using turbulent collision kernels derived from direct numerical simulations of droplet-laden turbulent flows. This in part is motivated by the recent study of Riemer and Wexler (2005, hereafter RW05), who solved the kinetic collection equation (KCE) using the turbulent collision kernel of ZWW01 (hereafter will be referred to as the ZWW-RW kernel). Wang et al. (2006a) pointed out several drawbacks and limitations of the ZWW-RW kernel, and questioned the relevance of the conclusions of RW05 to the growth of cloud droplets. Here we shall consider several improved versions of turbulent collision kernels appropriate for cloud droplets. We will compare different kernels and investigate their impact on the evolution of size distributions starting from an identical initial distribution.

\section{Turbulent collision kernels}

In this section, we introduce several formulations of turbulent collision kernels and discuss how the relevant physical mechanisms are included in these kernels. The Ayala kernel discussed in section $2 \mathrm{c}$ is the most realistic kernel in the context of cloud droplets. The ZWW-RW kernel contains several problems, which have been discussed in Wang et al. (2006a), and we consider it here to illustrate the quantitative differences on the resulting rate of droplet growth. Two alternative versions to the ZWW-RW kernel are also introduced in section $2 \mathrm{~b}$ : the mZWWnogr kernel is used to expose only the turbulent mechanism (without differential sedimentation effect); the mZWWgr kernel is designed to eliminate some of the problems in the ZWW-RW kernel. By using these different kernels, we shall gain a better understanding of the connection between the enhanced collision kernel by turbulence and its actual impact on the rate of droplet growth including 1) the sensitivity of the droplet growth to the nature and accuracy of the kernel, 2) the range of droplet sizes where turbulence enhancement can have the most impact on the droplet growth, and 3) the role of the turbulence enhancement relative to the gravitational mechanism. It will also enable us to develop a methodology which can identify different phases in the time evolution of the droplet growth. 
The Hall kernel (Hall 1980), a hydrodynamicalgravitational kernel without effects of air turbulence, will be used as a base case to compare the relative impact of turbulence. The Hall kernel is often used by the cloud physics community to study the growth of cloud droplets by collision-coalescence.

\section{a. The $Z W W-R W$ kernel}

The first turbulent collision kernel is a kernel developed by ZWW01 and was used by RW05 to study the growth of cloud droplets by turbulent collisioncoalescence. In ZWW01, the Stokes drag law was assumed and the gravitational settling was not considered. In RW05, the gravitational effect was treated separately by adding the Hall kernel to the parameterization of ZWW01.

In ZWW01, the turbulent geometric collision kernel in a bidisperse system was considered. It included the effect of turbulence on the relative velocity between two colliding droplets (the transport effect) and the nonuniform droplet distribution due to the interaction between particles and their surrounding airflow vortical structures (preferential concentration). Their model was based on the general kinematic formulation (e.g., Sundaram and Collins 1997; Wang et al. 2005b) of the geometric collision kernel in the form of

$$
K_{i j}^{g}=2 \pi R^{2}\left\langle\left|w_{r}\right|(r=R)\right\rangle g_{i j}(r=R),
$$

where the geometric collision radius $R$ is defined as $R=$ $r_{i}+r_{j}$, with $r_{i}$ and $r_{j}$ being the radii of the two colliding droplets; the superscript $g$ denotes geometric collision without consideration of local aerodynamic interactions of droplets; $w_{r}$ is the radial relative velocity between a particle pair of radii $r_{i}$ and $r_{j}$; and $g_{i j}$ is the radial distribution function. A list of symbols is compiled in appendix A. The average radial relative velocity $\left\langle\left|w_{r}\right|\right\rangle$ describes the turbulent transport effect, which is represented in ZWW01 as

$$
\left\langle\left|w_{r}\right|\right\rangle=\left[\frac{2}{\pi}\left(\left\langle w_{r, \text { accel }}^{2}\right\rangle+\left\langle w_{r, \text { shear }}^{2}\right\rangle\right)\right]^{1 / 2},
$$

where $\left\langle w_{r \text {,accel }}^{2}\right\rangle$ represents the contribution due to differential fluid acceleration and $\left\langle w_{r \text {,shear }}^{2}\right\rangle$ is the contribution due to local fluid shear. The shear term $\left\langle w_{r, \text { shear }}^{2}\right\rangle$ can be easily obtained using the theory of Saffman and Turner (1956), see also Wang et al. (1998a,b, 2005b) for further clarifications. The fluid acceleration term was parameterized by modifying the theory of Kruis and Kusters (1997) to match the direct simulation data of Wang et al. (2000) and ZWW01 for nonsettling particles.
Finally, the radial distribution function (RDF) at contact $g_{i j}(r=R)$ takes into account the preferential concentration effect and is modeled in terms of the RDFs for monodisperse particles $\left(g_{i i}\right.$ and $\left.g_{j j}\right)$ and a cross-correlation coefficient $\rho_{i j}$, as

$$
g_{i j}(R)=1.0+\rho_{i j}\left\{\left[g_{i i}(R)-1\right]\left[g_{j j}(R)-1\right]\right\}^{1 / 2},
$$

with the monodisperse RDFs and the coefficient $\rho_{i j}$ parameterized by empirically matching the direct numerical simulation (DNS) data of Wang et al. (2000) and ZWW01. The remaining details of the ZWW01 kernel are provided in appendix B.

RW05 adopted the above kernel for turbulent collision of nonsettling droplets. They then added the Hall kernel to it to account for the gravitational contribution as follows:

$$
K_{i j}=2 \pi R^{2}\left\langle\left|w_{r}\right|(r=R)\right\rangle g_{i j}(r=R) E_{i j}^{t}+\pi R^{2}\left|v_{s i}-v_{s j}\right| E_{i j}^{s} .
$$

RW05 set the collision efficiency $E_{i j}^{t}$ for the turbulent mechanism to one, but the collision efficiency $E_{i j}^{s}$ for the gravitational mechanism is assumed to be the same as that used in the Hall kernel (Hall 1980).

As pointed out recently by Wang et al. (2006a), there are a number of drawbacks in the above ZWW-RW kernel including 1) the rms fluctuation velocity of the air turbulence was overestimated by a factor of $\sqrt{3}, 2$ ) the radial distribution function was overestimated owing to the assumption of no sedimentation in ZWW01, and 3) several inconsistent treatments of the turbulent contribution as compared to the gravitational contribution, namely, the use of the Stokes drag and unity collision efficiency for the turbulent contribution.

\section{b. Modified $Z W W$ kernels}

We shall next introduce a modified turbulent kernel based on ZWW01. The parameterization is the same as that presented in the previous section for the turbulent contribution, but two modifications are introduced. The first is to replace the Stokes response time $\tau_{s}$ by a much more realistic inertial response time $\tau_{n}$ based on a nonlinear drag (Wang et al. 2006a) that would result in a realistic terminal velocity. The terminal velocity $v_{n}$ is now computed based on the following nonlinear drag law (Clift et al. 1978):

$$
m_{p}|\mathbf{g}|=6 \pi \mu r v_{n}\left[1+0.15\left(\frac{2 r \rho_{a} v_{n}}{\mu}\right)^{0.687}\right],
$$

where $m_{p}$ is the mass of the droplet, $\mathbf{g}$ is the gravitational acceleration. Wang et al. (2006a) demonstrated that the above equation yields a terminal velocity al- 
most identical to that used in the Hall kernel. The modified inertial response time $\tau_{n}$ is calculated as $\tau_{n}=$ $v_{n} /|\mathbf{g}|$. The second change is to use the correct rms fluctuation velocity of air turbulence, $u^{\prime}=2.02 \mathrm{~m} \mathrm{~s}^{-1}$ (see Wang et al. 2006a). The typical range of rms airflow fluctuation velocity in a given direction should be from 0.5 to $2.5 \mathrm{~m} \mathrm{~s}^{-1}$ according to observations (Weil and Lawson 1993; Smith and Jonas 1995; Furumoto et al. 2003). RW05 incorrectly used $u^{\prime}=3.5 \mathrm{~m} \mathrm{~s}^{-1}$, a factor of $\sqrt{3}$ too larger than what should have been assumed [e.g., see reply by Riemer and Wexler (2006)].
Two versions of the modified ZWW kernel will be considered here. The first version only considers the turbulent contribution with the above two modifications and neglects the gravitational effect. This kernel will be referred to as the modified ZWW kernel without gravity, or mZWWnogr for short. The same collision efficiency as in the Hall kernel is applied to the mZWWnogr kernel.

The second version includes the gravitational contribution by adding to the above mZWWnogr kernel the Hall kernel as follows:

$$
K_{i j}=2 \pi R^{2} E_{i j}^{s} \times\left\{\frac{2}{\pi}\left[\left(\left\langle w_{r, \text { accel }}^{2}\right\rangle+\left\langle w_{r, \text { shear }}^{2}\right\rangle\right) g_{i j}^{2}(r=R)+\frac{\pi}{8}\left(v_{n i}-v_{n j}\right)^{2}\right]\right\}^{1 / 2} .
$$

This second version will be called the modified ZWW kernel with gravity, or mZWWgr for short. Note that the above formulation in combining the turbulent mechanism and the gravitational mechanism follows closely the studies of Wang et al. (1998b) and Dodin and Elperin (2002).

\section{c. The Ayala kernel}

Even with the above modifications, the radial distribution function in the above kernels is overestimated, as the differential sedimentation will reduce the crosscorrelation $\rho_{i j}$ as well as the interaction time of droplets with turbulent vortical structure (A05; Wang et al. 2006a). Very recently, A05 developed a kernel based on direct simulations of turbulent collisions of sedimenting droplets. His study of the geometric collision kernel considered simultaneously the effects of air turbulence and gravity. A05 also considered the hydrodynamic interactions in a turbulent air, using a hybrid DNS approach (Ayala et al. 2007). Since the data on collision efficiency have not been analyzed to the point when a parameterization can be developed, in our preliminary study here we shall only consider the effects of turbulence on the geometric collision kernel. The same collision efficiency as in the Hall kernel and the terminal velocity $v_{n}$ based on the nonlinear drag are employed. The additional impact on droplet growth due to enhanced collision efficiency by turbulence (Wang et al. 2005a) will be studied in the future when an accurate parameterization of the enhanced collision efficiency becomes available.

Following Dodin and Elperin (2002), A05 decomposes the radial relative velocity between two droplets into a random part $\xi$ caused by turbulent fluctuations and a deterministic part $h$ due to gravity. For a given angle of contact $\alpha$ defined as the angle between the line connecting the droplet centers and the vertical axis, the deterministic part can be expressed as $h=\left|v_{n i}-v_{n j}\right|$ $\cos \alpha$. The random part has a mean zero and a standard deviation denoted by $\sigma$. After averaging over all contact angles, the radial relative velocity $\left\langle\left|w_{r}\right|\right\rangle$ is expressed as (Dodin and Elperin 2002; A05)

$$
\left\langle\left|w_{r}\right|\right\rangle=\sqrt{\frac{2}{\pi}} \sigma\left[\frac{1}{2} \sqrt{\pi}\left(b+\frac{0.5}{b}\right) \operatorname{erf}(b)+\frac{1}{2} \exp \left(-b^{2}\right)\right],
$$

where the parameter $b$ is defined as

$$
b=\frac{\left|v_{n i}-v_{n j}\right|}{\sigma \sqrt{2}} .
$$

The key is then to determine the turbulent contribution to the relative motion in the radial direction. The variance of this relative fluctuation is expressed as

$$
\sigma^{2}=\left\langle\left(v^{\prime(i)}\right)^{2}\right\rangle+\left\langle\left(v^{\prime(j)}\right)^{2}\right\rangle-2\left\langle\left(v^{\prime(i)} \boldsymbol{v}^{\prime(j)}\right)\right\rangle,
$$

where $v^{\prime(i)}$ and $v^{\prime(j)}$ are the velocity fluctuation of the two colliding droplets in the radial direction. Assuming that the terminal velocities dominate the relative motion of droplets right before collision, A05 developed a leading-order approximation to the velocity variances, $\left\langle\left(v^{\prime(i)}\right)^{2}\right\rangle$ and $\left\langle\left(v^{\prime(j)}\right)^{2}\right\rangle$, and cross variance $\left\langle\left(v^{\prime(i)} v^{\prime(j)}\right)\right\rangle$ in terms of droplet response time, terminal velocity, and space-time velocity correlations of the turbulent flow, using the integral form of the equation of motion for droplets. The resulting expressions for the velocity variances, taken from A05, are provided in appendix C. A05 showed that his analytical expression for radial relative velocity agrees with direct simulation data for most cases relevant to cloud droplets.

A05 also considered the modeling of RDF for sedimenting droplets by fitting the recent theory of 
Chun et al. (2005) to his DNS data. The RDF at contact $g_{i j}(r=R)$ is written as

$$
g_{i j}(r=R)=\left(\frac{\eta^{2}+r_{c}^{2}}{R^{2}+r_{c}^{2}}\right)^{C_{1} / 2},
$$

where $r_{c}$ is a distance below which the fluid acceleration-driven diffusion predominates the ordered relative drifting due to preferential concentration (Chun et al. 2005). By curve fitting DNS data of RDF, empirical expressions for the exponent $C_{1}$ and the transition distance $r_{c}$ are obtained. These are also presented in appendix C.

In summary, the A05 parameterization of the turbulent collision kernel is the most realistic and comprehensive kernel available when applied to cloud droplets, although the effect of turbulence on collision efficiency is not yet included.

\section{d. Comparison of different kernels}

In this section, we compare the magnitudes of the five collection kernels we have introduced, namely, 1) the Hall kernel, 2) the ZWW-RW kernel, 3) the mZWWnogr kernel, 4) the mZWWgr kernel, and 5) the Ayala kernel. The unit for the kernels in all plots to be discussed is $\mathrm{cm}^{3} \mathrm{~s}^{-1}$.

Figure 1 is a contour plot of the Hall kernel for different droplet-droplet size combinations. The contour plot is symmetric with respect to the $45^{\circ}$ line on which the Hall kernel becomes zero as only gravitational settling is considered here. The magnitude of the Hall kernel can vary by over 10 orders of magnitude when the droplet size varies from $1 \mu \mathrm{m}$ to $1 \mathrm{~mm}$ due to the large changes in both collision efficiency and the terminal velocity. The Hall kernel is less than $0.03 \mathrm{~cm}^{3} \mathrm{~s}^{-1}$ for droplets less than $100 \mu \mathrm{m}$. For droplets larger than $100 \mu \mathrm{m}$ in radius, the kernel is proportional to the differential sedimentation velocity, as the collision efficiency is close to one. If the larger droplet in the colliding pair is $1 \mathrm{~mm}$ in radius, the kernel is around 20 $\mathrm{cm}^{3} \mathrm{~s}^{-1}$.

The contour plot for the ZWW-RW kernel is shown in Fig. 2. As noted by RW05, the turbulent contribution dominates the ZWW-RW kernel for droplet pairs whose sizes fall between 50 and about $500 \mu \mathrm{m}$. For example, the ZWW-RW kernel can be around 5.0 $\mathrm{cm}^{3} \mathrm{~s}^{-1}$ when the larger droplet in the pair is $100 \mu \mathrm{m}$, which is larger than the Hall kernel by two orders of magnitude. For droplets smaller than $50 \mu \mathrm{m}$, the turbulent contribution is also much larger than the gravitational contribution due to the assumed unity collision efficiency for the former, but the hydrodynamicgravitational collision efficiency for the latter.
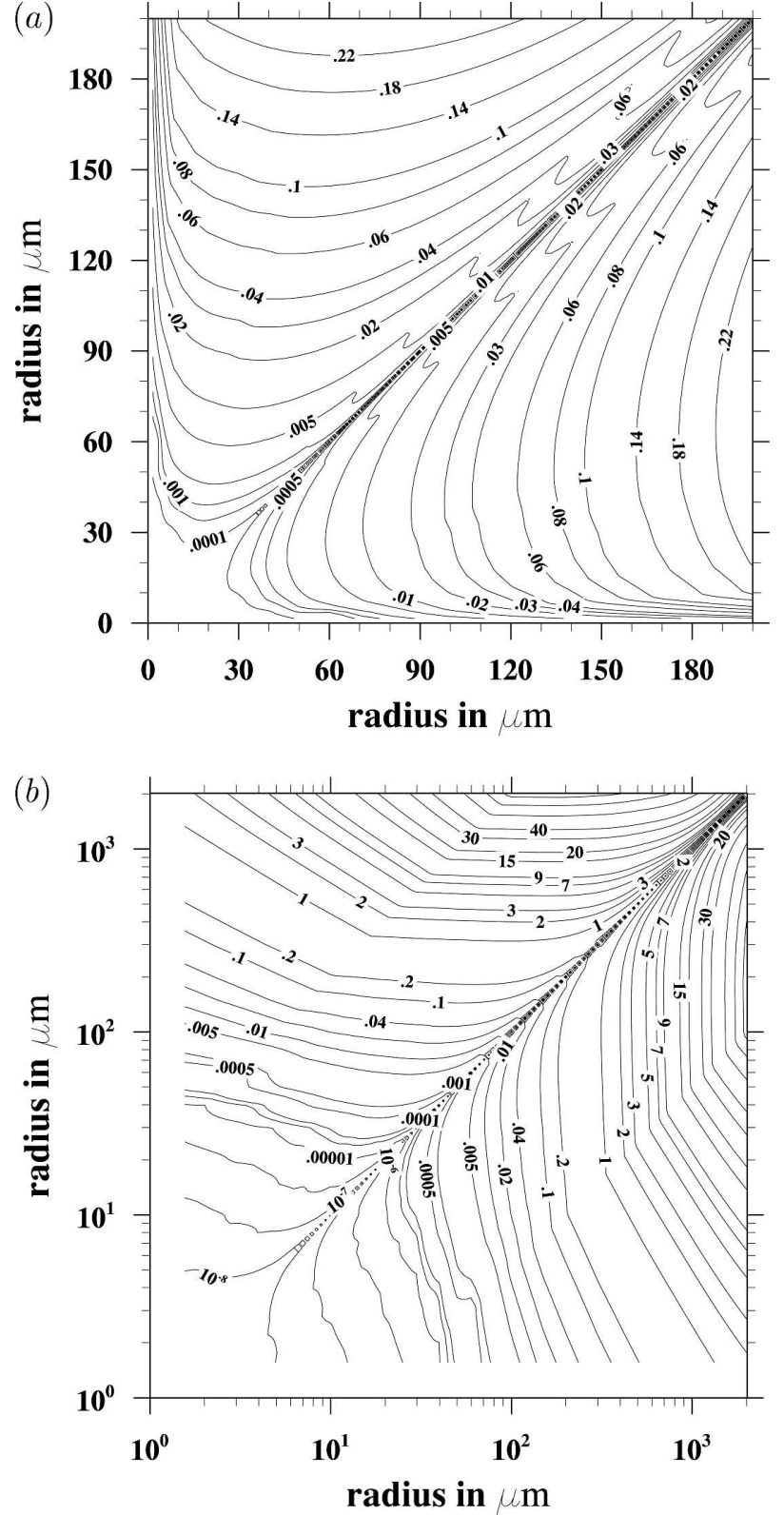

FIG. 1. Contour plots of the Hall kernel for (a) linear size axes up to $200 \mu \mathrm{m}$ and (b) logarithmic size axes up to $2 \mathrm{~mm}$.

Berry and Reinhardt (1974) proposed three phases of growth of cloud droplets by collision-coalescence. After dividing the size spectrum into small cloud droplets (S1, roughly less than $50 \mu \mathrm{m}$ ) and larger drops (S2, roughly larger than $50 \mu \mathrm{m}$ ), Berry and Reinhardt (1974) showed that the initial growth is governed by S1-S1 autoconversion to add water to S2, followed by accretion via S2-S1 interactions, and eventually by $\mathrm{S} 2-$ S2 large hydrometeor self-collection. The contour plot (Fig. 2) implies that both the autoconversion and ac- 

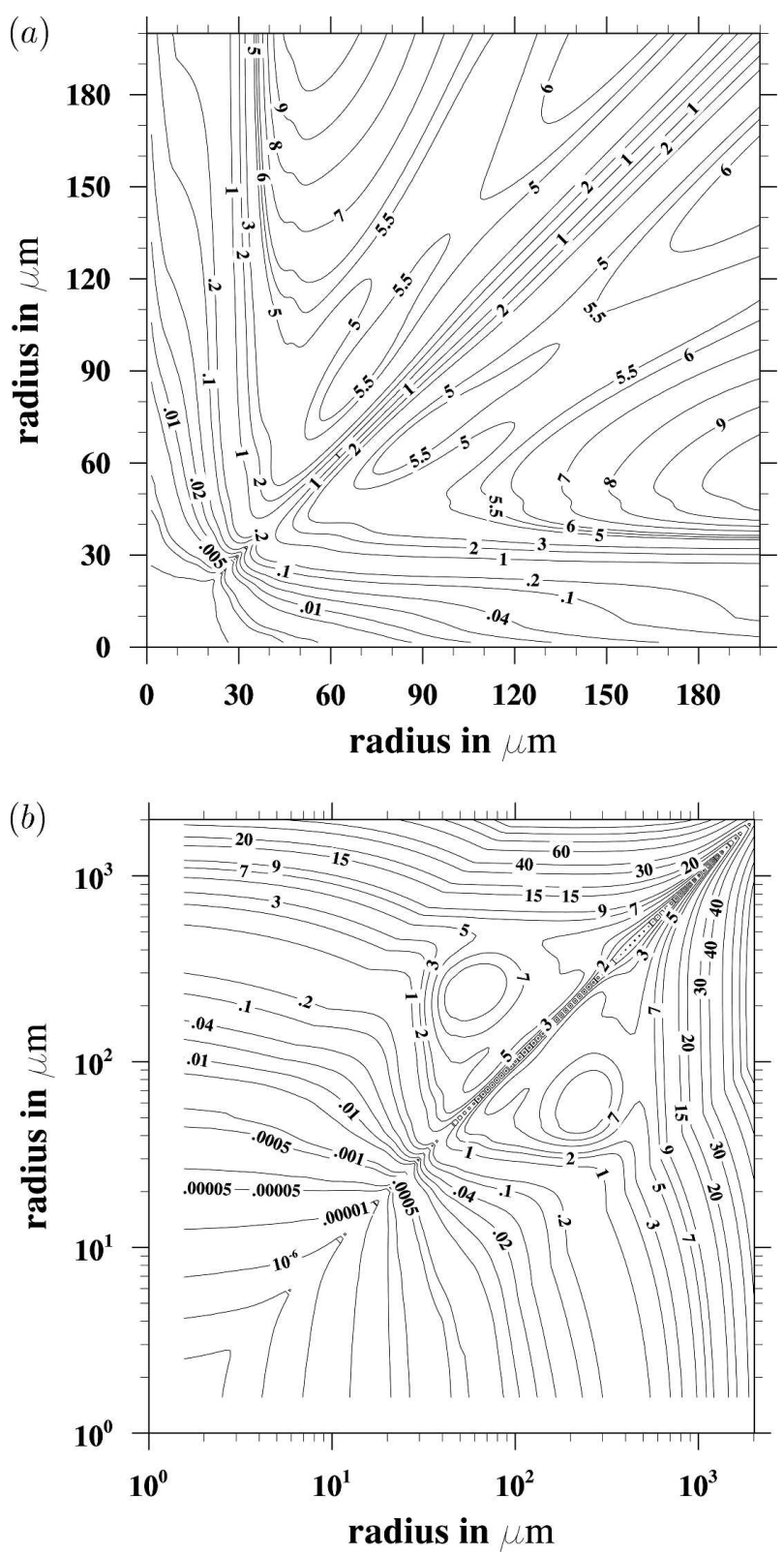

FIG. 2. Contour plots of the ZWW-RW kernel for (a) linear size axes up to $200 \mu \mathrm{m}$ and (b) logarithmic size axes up to $2 \mathrm{~mm}: \varepsilon=$ $300 \mathrm{~cm}^{2} \mathrm{~s}^{-3}$ and $u^{\prime}=350 \mathrm{~cm} \mathrm{~s}^{-1}$ as in RW05.

cretion rates are dramatically increased by the turbulent contribution in the ZWW-RW kernel, as will be shown in section 3 .

The contour plots for the mZWWnogr kernel and the mZWWgr kernel are displayed in Figs. 3 and 4, respectively. The mZWWnogr kernel is in general smaller than the ZWW-RW kernel for droplets less than 100 $\mu \mathrm{m}$ owing to the use of the nonlinear drag and the correct fluid rms fluctuation velocity. For example, the mZWWnogr kernel is about half the value of the
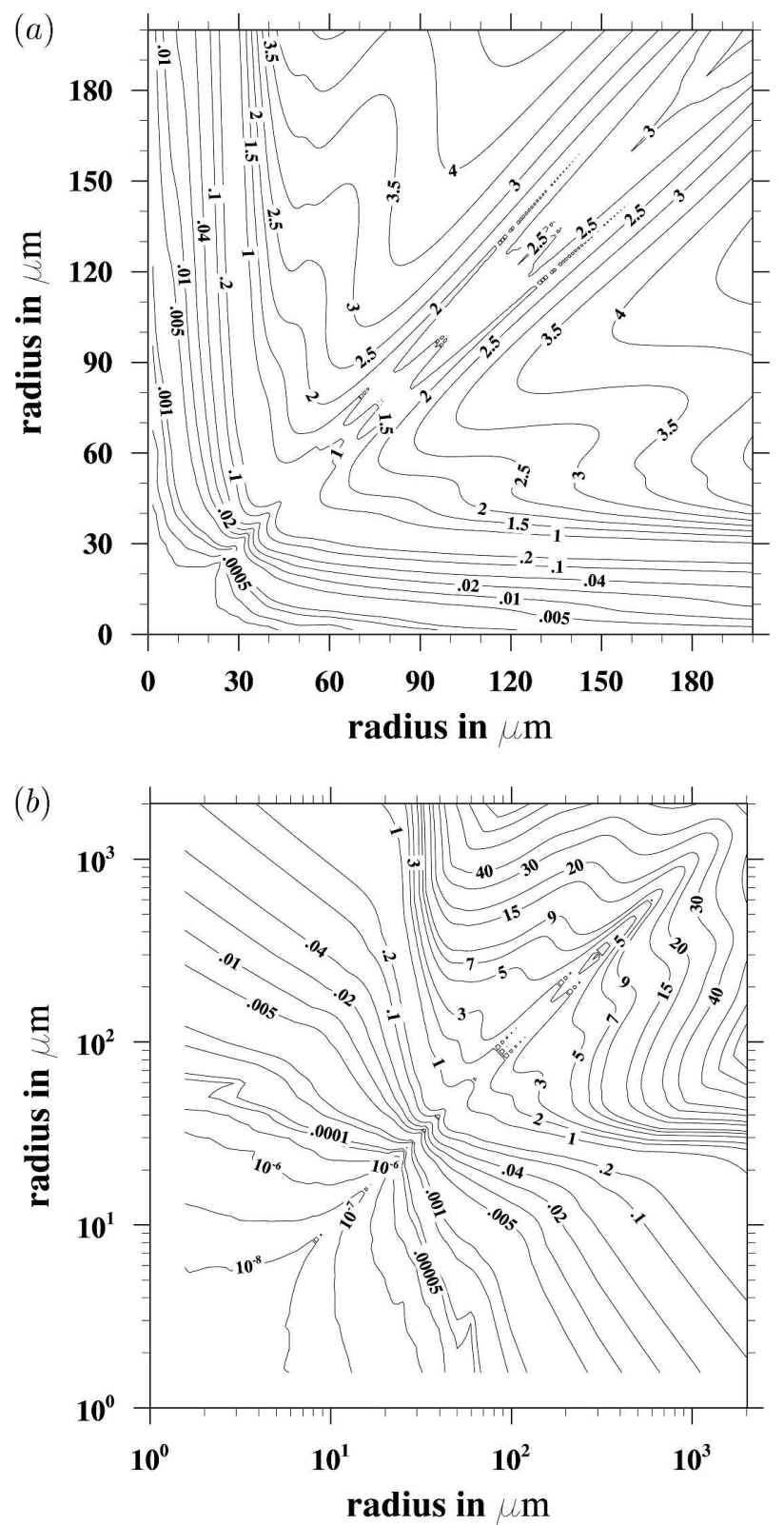

FIG. 3. Contour plots of the mZWWnogr kernel for (a) linear size axes up to $200 \mu \mathrm{m}$ and (b) logarithmic size axes up to $2 \mathrm{~mm}$ : $\varepsilon=300 \mathrm{~cm}^{2} \mathrm{~s}^{-3}$ and $u^{\prime}=202 \mathrm{~cm} \mathrm{~s}^{-1}$.

ZWW-RW kernel for droplets pairs whose larger droplet is $100 \mu \mathrm{m}$ in radius. However, the mZWWnogr kernel is larger than the ZWW-RW kernel for drops of the order of $1 \mathrm{~mm}$, as a result of the much slower decay of RDF with increasing droplet size due to the nonlinear drag (see Fig. 5). In fact, RDF may remain much larger than one when the terminal velocity reaches a constant value for droplets larger than $2 \mathrm{~mm}$ in radius (which also implies a constant effective inertial response time). This slow decay is physically incorrect since the model 


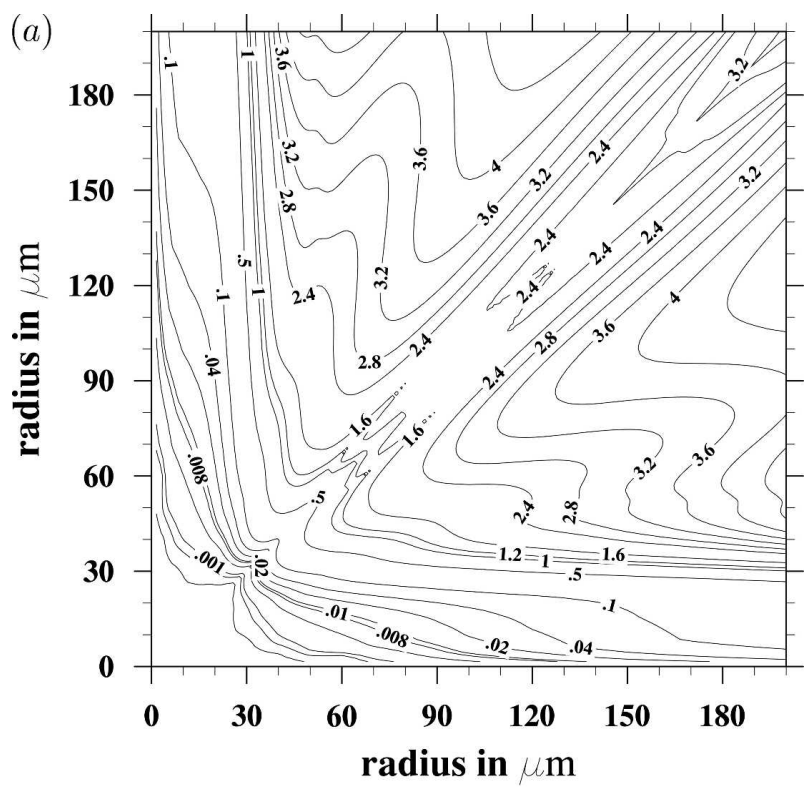

(b)

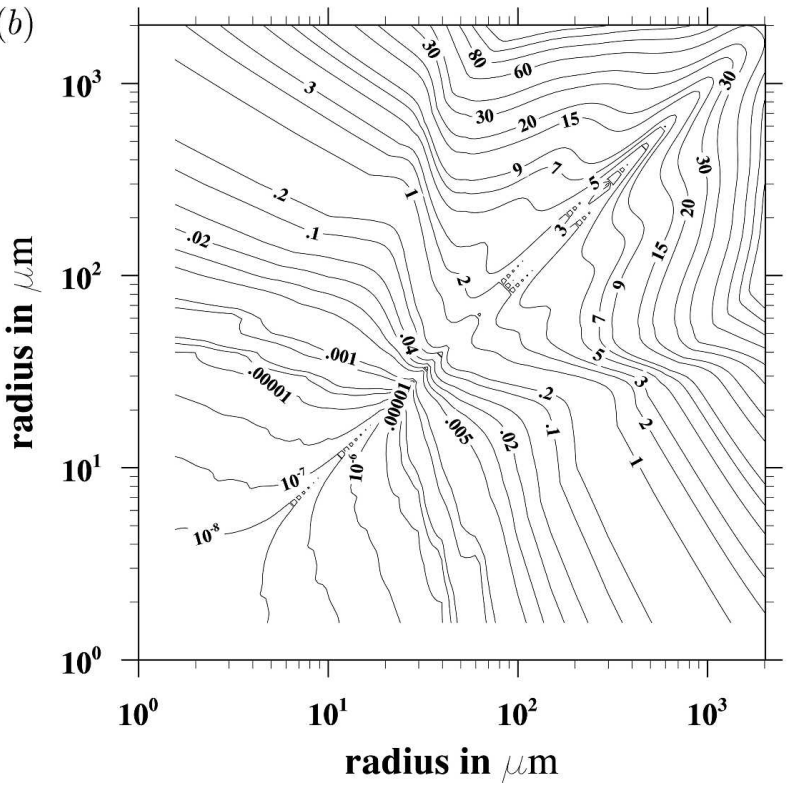

FIG. 4. Contour plots of the mZWWgr kernel for (a) linear size axes up to $200 \mu \mathrm{m}$ and (b) logarithmic size axes up to $2 \mathrm{~mm}$ : $\varepsilon=$ $300 \mathrm{~cm}^{2} \mathrm{~s}^{-3}$ and $u^{\prime}=202 \mathrm{~cm} \mathrm{~s}^{-1}$.

for RDF here was derived from ZWW01 without the influence of sedimentation (e.g., Wang et al. 2006a).

The mZWWgr kernel is very similar to the mZWWnogr kernel for most size combinations except that, by definition, the mZWWgr kernel is always larger than the Hall kernel. While the gravitational mechanism dominates the kernel, the air turbulence is effective for small cloud droplets (less than $50 \mu \mathrm{m}$ ) due to the relatively small magnitude of the gravitational kernel. Therefore, both the autoconversion and the accre-

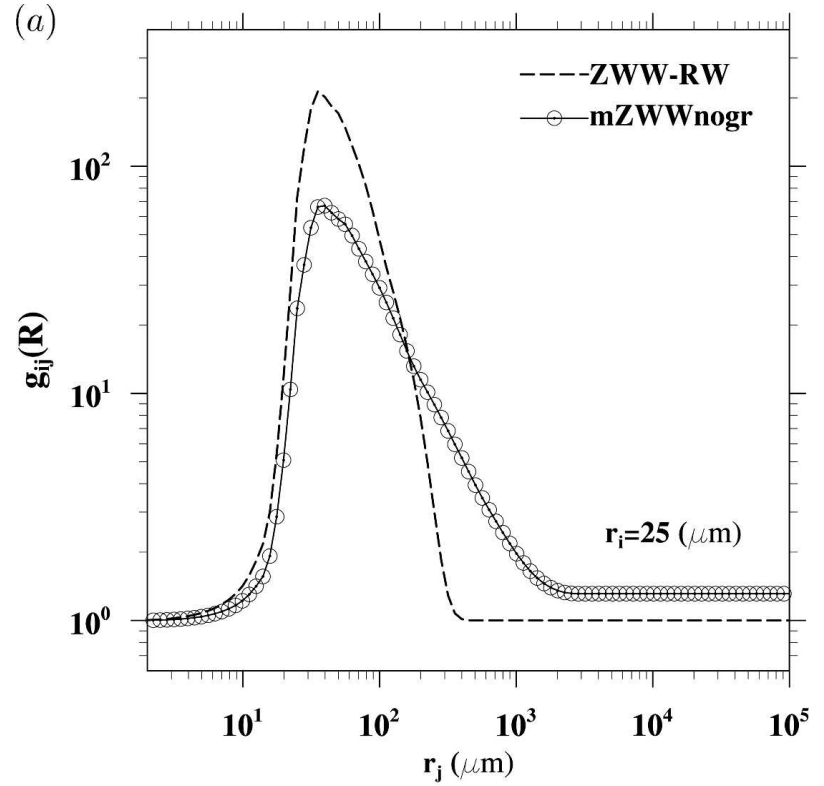

(b)

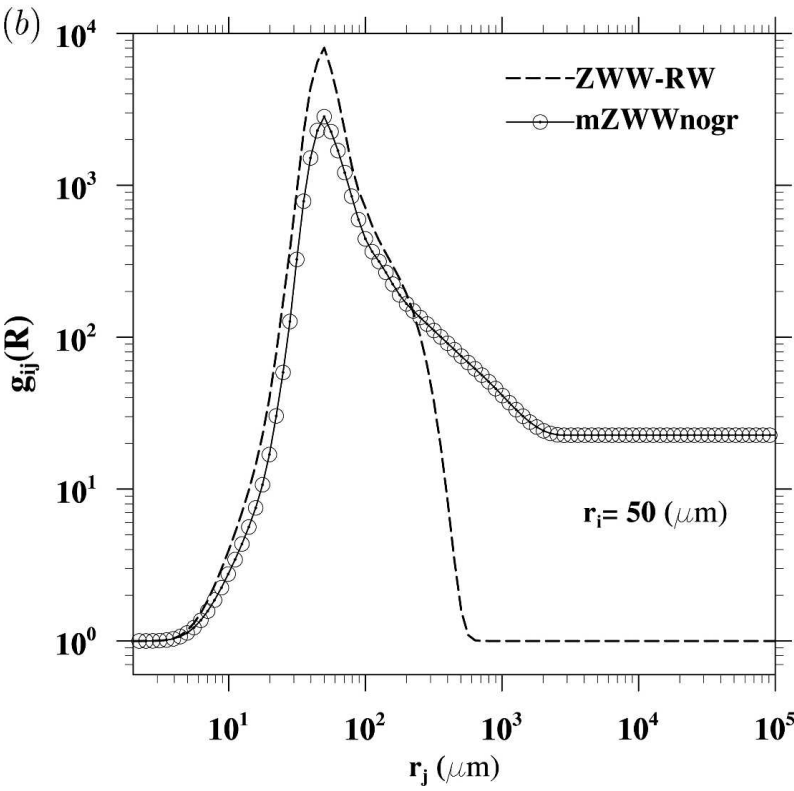

FIG. 5. Comparison of radial distribution function $g_{i j}(R)$ in the mZWWnogr kernel and in the ZWW-RW kernel for two different $r_{i}: g_{i j}(R)$ in the mZWWnogr kernel is calculated based on the nonlinear drag force with $\varepsilon=300 \mathrm{~cm}^{2} \mathrm{~s}^{-3}$ and $u^{\prime}=202 \mathrm{~cm} \mathrm{~s}^{-1}$, while $g_{i j}(R)$ in the ZWW-RW kernel is calculated based on the Stokes drag with $\varepsilon=300 \mathrm{~cm}^{2} \mathrm{~s}^{-3}$ and $u^{\prime}=350 \mathrm{~cm} \mathrm{~s}^{-1}$.

tion phase for the mZWWgr kernel are slightly more effective than for the Hall kernel.

The contour plot for the Ayala kernel is shown in Fig. 6. Compared to the other turbulent collection kernels considered above, the Ayala kernel shows a much less dramatic enhancement by air turbulence, but at the same time the enhancement appears for all droplets less 


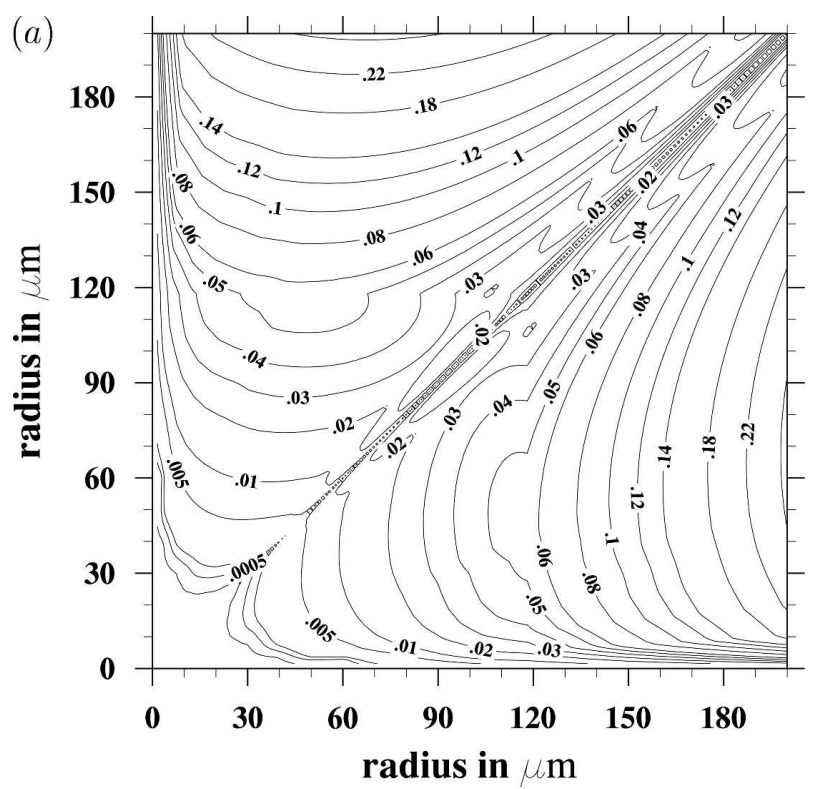

(a)

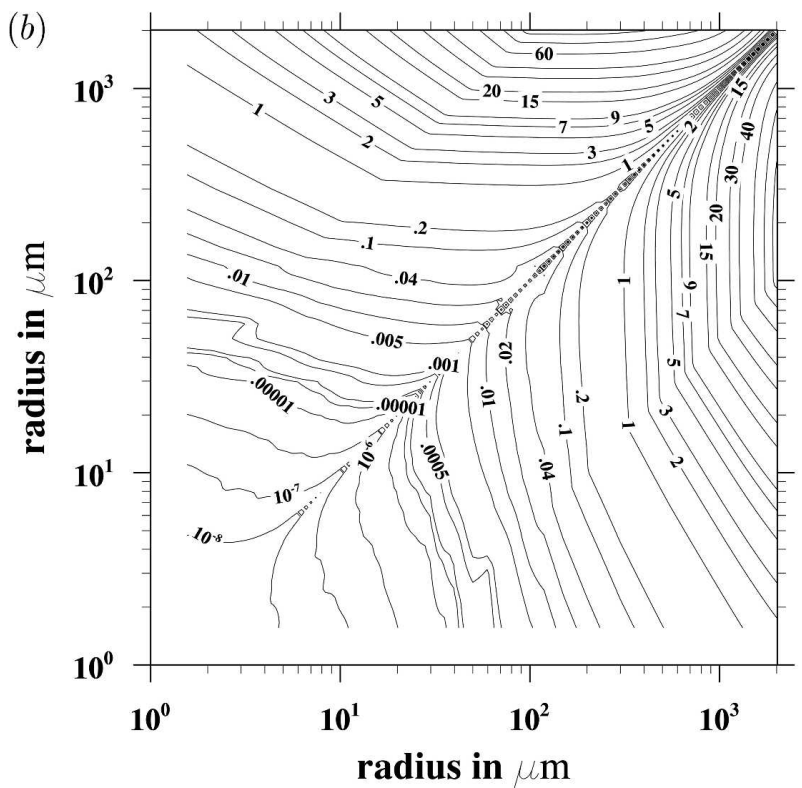

FIG. 6. Contour plots of the Ayala kernel for (a) linear size axes up to $200 \mu \mathrm{m}$ and (b) logarithmic size axes up to $2 \mathrm{~mm}: \varepsilon=300$ $\mathrm{cm}^{2} \mathrm{~s}^{-3}$ and $u^{\prime}=202 \mathrm{~cm} \mathrm{~s}^{-1}$.

than $100 \mu \mathrm{m}$. The effect of air turbulence is negligible for droplets larger than $100 \mu \mathrm{m}$ in the Ayala kernel, as a result of the assumed vanishing preferential concentration in the Ayala kernel for droplets larger than 100 $\mu \mathrm{m}$ due to the effect of strong sedimentation. The distribution of RDF over droplet size in the Ayala kernel is much more localized than that in the other turbulent kernels; see for example, Fig. 7 for the mZWWnogr kernel as compared to Fig. 8 for the Ayala kernel.

We would like to point out that the turbulent contri-
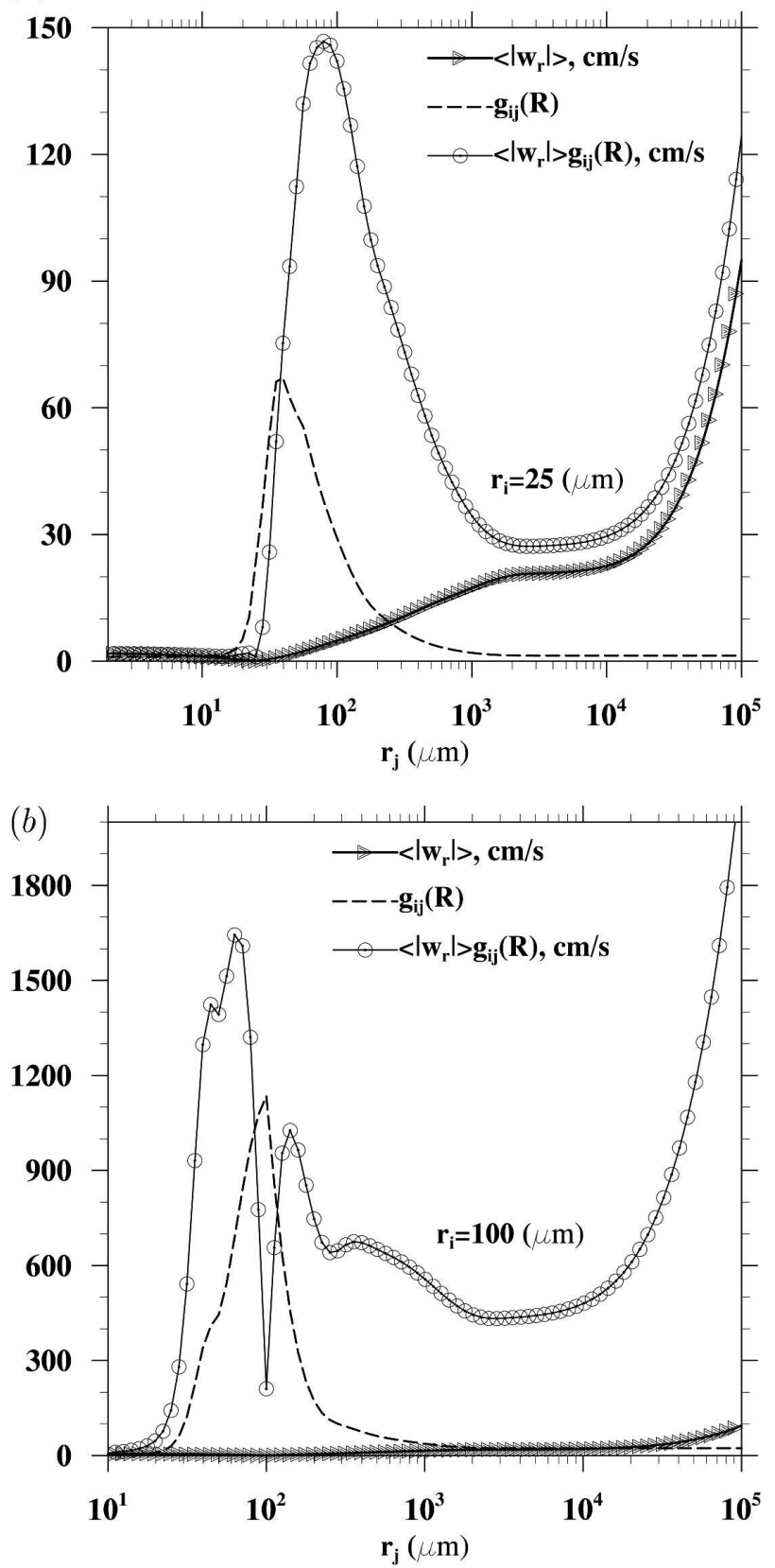

FIG. 7. The radial relative velocity and the radial distribution function as a function of $r_{j}$ with (a) $r_{i}=25$ and (b) $r_{i}=100 \mu \mathrm{m}$ in the mZWWnogr kernel: $\varepsilon=300 \mathrm{~cm}^{2} \mathrm{~s}^{-3}$ and $u^{\prime}=202 \mathrm{~cm} \mathrm{~s}^{-1}$.

bution in the ZWW-RW, mZWWnogr, and mZWWgr kernels contains the effect of preferential concentration as quantified by RDF, which tend to create an internal region with a maximum local collection kernel. For example, in Fig. 2 for the ZWW-RW kernel, this maximum happens when $100<r_{i}<400 \mu \mathrm{m}$ and $r_{j} \approx 50 \mu \mathrm{m}$. In the case of the mZWWnogr kernel shown in Fig. 3a, 

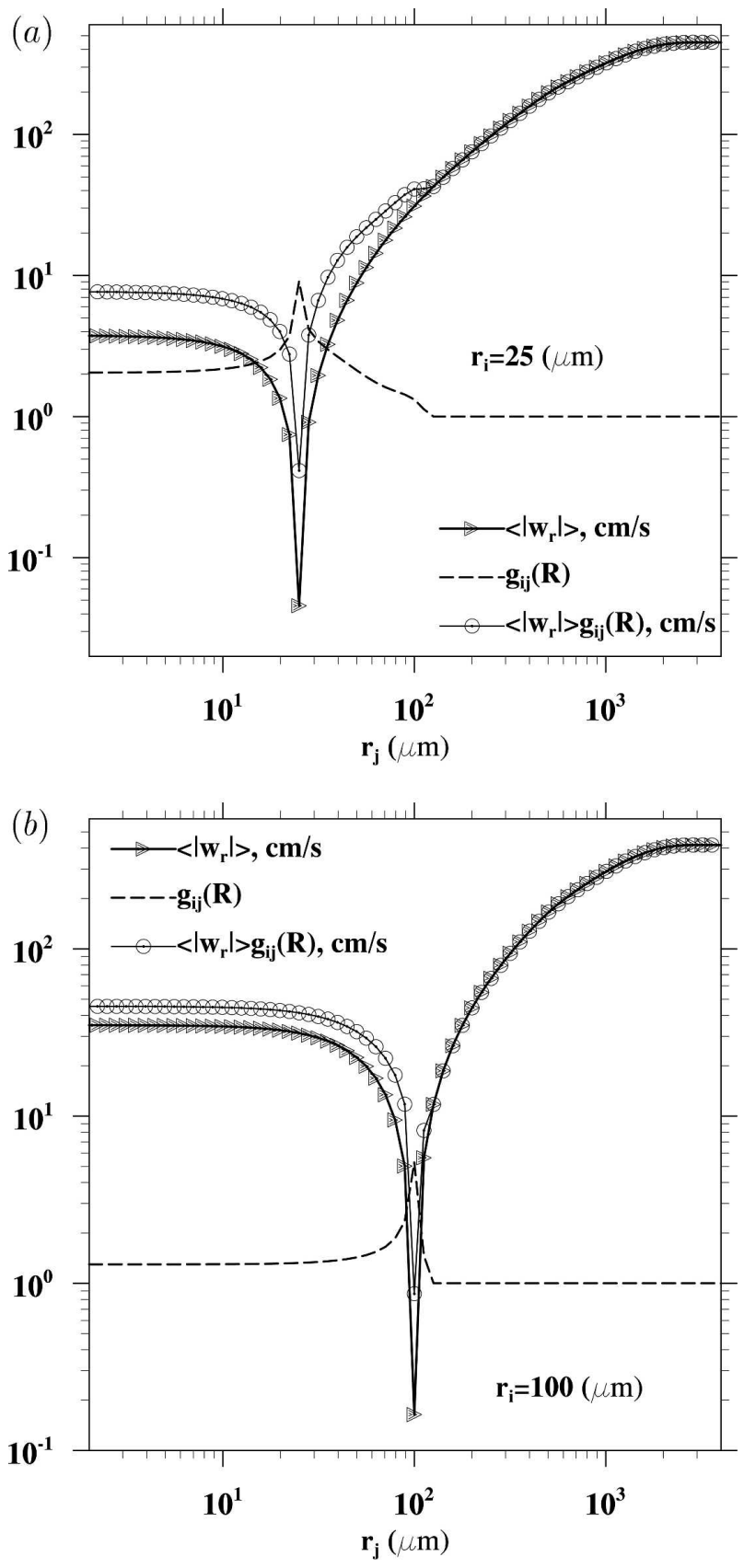

FIG. 8. The radial relative velocity and the radial distribution function in the Ayala kernel as a function of $r_{j}$ with (a) $r_{i}=25$ and (b) $r_{i}=100 \mu \mathrm{m}: \varepsilon=300 \mathrm{~cm}^{2} \mathrm{~s}^{-3}$ and $u^{\prime}=202 \mathrm{~cm} \mathrm{~s}^{-1}$.

the maximum occurs along two tilted lines with angles at about $10^{\circ}$ and $30^{\circ}$. Similar internal regions are found in the mZWWgr kernel shown in Fig. 4a, but with smaller angles due to the addition of the gravitational mechanism. The Ayala kernel also shows the maxima along the line of about $10^{\circ}$ to $15^{\circ}$ angle. This type of internal local maxima is a result of the combined effect of RDF and the radial relative velocity. The maximum in RDF tends to occur for droplet pairs of similar sizes or along the $45^{\circ}$ line in the contour plots, while the radial relative velocity obtains its maximum along the $0^{\circ}$ line in the contour plots. The internal maximum regions would not occur if the preferential concentration is not present, such as in the Hall kernel. As a further demonstration of this, we plot in Figs. 7 and 8 the RDF, radial relative velocity, and their product as a function of $r_{j}$ with two fixed values of $r_{i}$, for the mZWWnogr kernel and the Ayala kernel, respectively. The plots show that the peak in RDF could cause local maxima in the product at locations shifted from the peak location of the RDF.

An important difference between the Hall kernel and the turbulent kernels is a nonzero value on the diagonal in the latter case; namely, droplets of equal size can collide due to the turbulent mechanism.

As a summary, Fig. 9 displays contour plots for the ratio of each turbulent collision kernel over the Hall kernel. This ratio measures the level of enhancement by the air turbulence, relative to the gravitational collision mechanism. The relative ratio for the mZWWgr kernel and that of the mZWWnogr kernel are rather similar (see Figs. 9b,c) except that the former is always greater than one. The mZWWnogr kernel is larger than the Hall kernel if both colliding droplets are larger than $30 \mu \mathrm{m}$; see Fig. 9b. If one of droplets in the pair is less than $30 \mu \mathrm{m}$, however, the mZWWnogr is less than the Hall kernel. This implies that both the autoconversion from droplets less than $30 \mu \mathrm{m}$ and the capturing of small cloud droplets by large drops through accretion (Berry and Reinhardt 1974) for the mZWWnogr kernel is slower than in the Hall kernel.

The ZWW-RW kernel has a much greater relative ratio than that of the mZWWgr kernel. One reason for this is the assumed unit collision efficiency for the turbulent mechanism in the ZWW-RW kernel, while the mZWWgr kernel uses the collision efficiency in the Hall kernel. Another reason is the different $u^{\prime}$ values used in these two different cases. Overall, the ZWW-RW kernel overestimates the turbulent contribution, relative to the gravitational contribution, by one to two orders of magnitude in most regions when compared to the Ayala kernel. Compared with the other three turbulent collision kernels, the Ayala kernel has a moderate enhancement with a relative ratio less than 2.0 for most regions. Furthermore, when one of the droplets in the pair is larger than $100 \mu \mathrm{m}$, the Ayala kernel is very close to the Hall kernel. However, the Ayala kernel could be larger than the mZWWgr kernel when one of the droplets in the pair is less than $30 \mu \mathrm{m}$, owing to a coupling mechanism between the relative motion due 

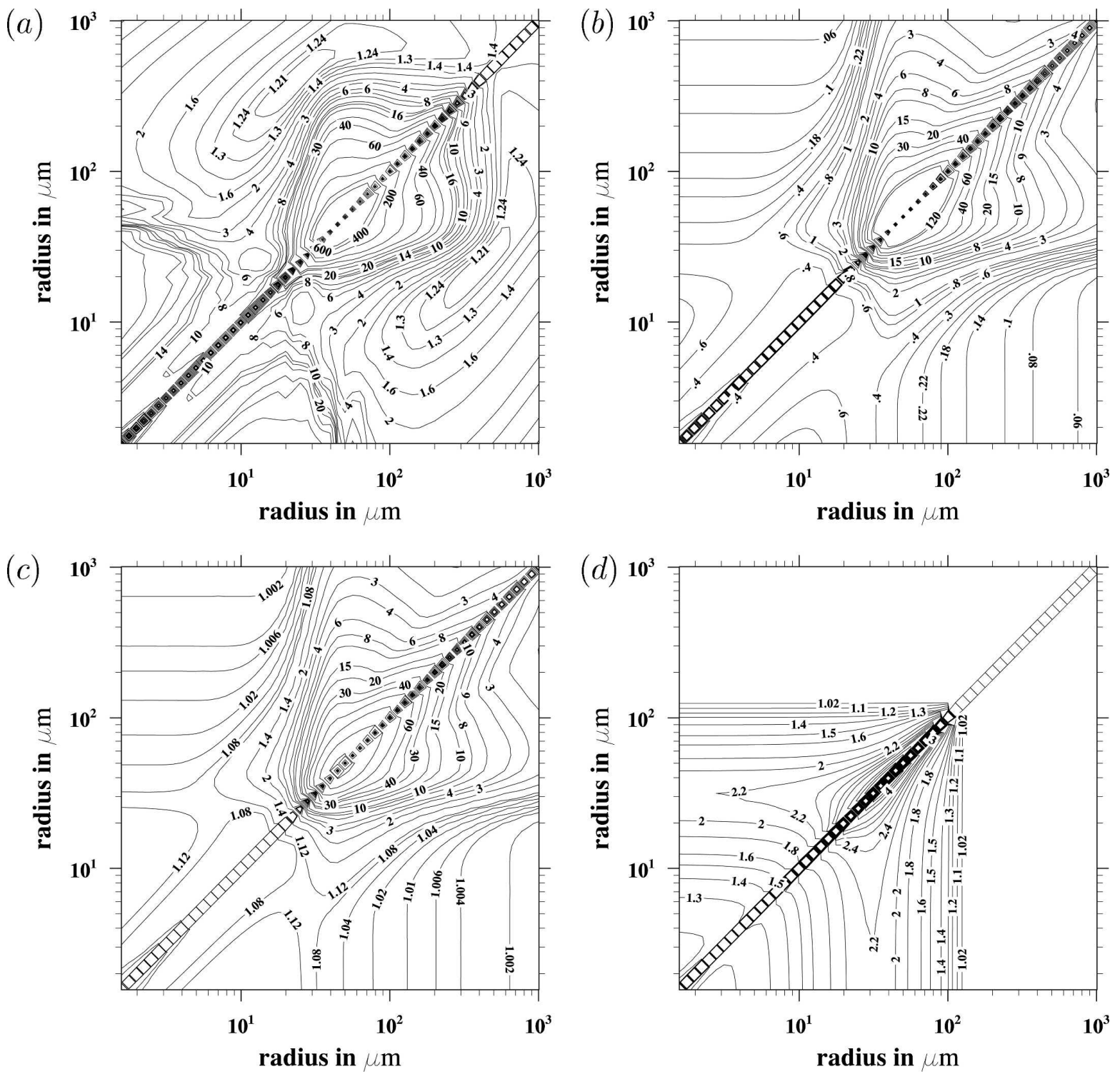

FIG. 9. Contour plots of the ratio of each turbulent collision kernel to the Hall kernel: (a) the ZWW-RW kernel, (b) the mZWWnogr kernel, (c) the mZWWgr kernel, and (d) the Ayala kernel.

to differential sedimentation and the relative motion due to air turbulence (A05).

To further illustrate the different levels of enhancement, we divide the droplet size into three groups: droplets less than $50 \mu \mathrm{m}$ in radius (A group), from 50 to $100 \mu \mathrm{m}$ (B group), and larger than $100 \mu \mathrm{m}$ (C group). Then there are six pair size combinations (or zones): $\mathrm{AA}, \mathrm{AB}, \mathrm{AC}, \mathrm{BB}, \mathrm{BC}$, and CC. For example, AB represents the case when $a_{j} \leq 50 \mu \mathrm{m}$ and $50 \leq a_{i} \leq 100 \mu \mathrm{m}$. Table 1 lists the maximum and minimum values of each turbulent kernel when normalized by the Hall kernel for each zone, and the actual size combinations where the maximum and minimum values occur. For example, for the case of Ayala kernel in the $\mathrm{AB}$ zone, a maximum ratio of 6.61 occurs at $a_{i}=50$ and $a_{j}=47.2 \mu \mathrm{m}$, and a minimum ratio of 1.31 occurs at $a_{i}=100$ and $a_{j}=$ $2.34 \mu \mathrm{m}$. The radius grid used to define the collection kernel is based on a geometric grid with $a_{i}=a_{1} \times 2^{i / 12}$. The line $a_{i}=a_{j}$ is excluded since the Hall kernel is zero when the two colliding droplets are of the same size. Several important observations can be made. The maximum values in the ZWW-RW kernel are at least two orders of magnitude larger than the more realistic Ayala kernel. In most cases, the maximum values occur for nearly equal-size droplets implying the importance of the preferential concentration effect. The maximum 
TABLE 1. The ratio of turbulent collection kernel to the Hall kernel: the maximum and minimum and the corresponding droplet sizes in each zone.

\begin{tabular}{lcccccc}
\hline \hline Kernel & AA & AB & AC & BB & BC & CC \\
\hline Ayala & $7.12,1.17$ & $6.61,1.31$ & $1.45,1.01$ & $6.32,1.45$ & $3.04,1.01$ & $2.39,1.01$ \\
& $(42.0,39.7)$ & $(50.0,47.2)$ & $(100.0,50.0)$ & $(53.0,50.0)$ & $(100.0,94.4)$ & $(106.0,100.0)$ \\
& $(2.48,1.56)$ & $(100.0,2.34)$ & $(2136.0,1.66)$ & $(100.0,50.0)$ & $(2136.0,50.0)$ & $(2136.0,100.0)$ \\
mZWWnogr & $558.2,0.28$ & $547.0,0.17$ & $62.9,0.05$ & $513.7,62.9$ & $173.7,1.08$ & $151.4,0.49$ \\
& $(47.2,44.5)$ & $(50.0,47.2)$ & $(100.0,50.0)$ & $(53.0,50.0)$ & $(100.0,94.4)$ & $(106.0,100.0)$ \\
mZWWgr & $(50.0,2.48)$ & $(100.0,3.94)$ & $(2136.0,14.0)$ & $(100.0,50.0)$ & $(2136.0,100.0)$ & $(2136.0,377.6)$ \\
& $558.2,1.04$ & $547.0,1.01$ & $63.0,1.00$ & $513.7,63.0$ & $173.7,1.47$ & $151.4,1.11$ \\
& $(47.2,44.5)$ & $(50.0,47.2)$ & $(100.0,50.0)$ & $(53.0,50.0)$ & $(100.0,94.4)$ & $(106.0,100.0)$ \\
ZWW-RW & $(50.0,2.48)$ & $(100.0,3.94)$ & $(2136.0,11.8)$ & $(100.0,50.0)$ & $(2136.0,100.0)$ & $(2136.0,377.6)$ \\
& $1948.5,1.89$ & $1764.2,1.34$ & $163.3,1.19$ & $1557.8,159.5$ & $339.2,1.21$ & $223.9,1.23$ \\
& $(42.0,39.7)$ & $(50.0,44.5)$ & $(100.0,50.0)$ & $(56.1,50.0)$ & $(100.0,89.1)$ & $(112.0,100.0)$ \\
& $(50.0,5.26)$ & $(100.0,8.84)$ & $(336.0,17.7)$ & $(100.0,56.1)$ & $(635.0,50.0)$ & $(673.0,100.0)$ \\
\hline
\end{tabular}

values cited in Table 1 tend to be localized regions in Fig. 9, while the minimum values cited in Table 1 are probably more representative of the overall enhancement by air turbulence in each zone. The Ayala kernel actually has a larger minimum value in zone AA and zone $\mathrm{AB}$ than the mZWWgr kernel. We will show in the next section that this moderate but more persistent enhancement in the Ayala kernel over the small to medium droplet size range can have a significant impact on the early stage of droplet growth by autoconversion.

In the following, most results from the kinetic collection equations will be presented with the Ayala kernel. Other kernels will be used to help understand the sensitivity of the rate of droplet growth to the nature and accuracy of the kernel, the different phases of the collision-coalescence growth, and the precise role of turbulence enhancement.

\section{Growth of cloud droplets}

Here we examine the droplet size distributions at different times and compare the results for the five different kernels discussed in the last section. The following initial distribution,

$$
n(x, t=0)=\frac{N_{0}}{\bar{x}_{f 0}} \exp \left(-\frac{x}{\bar{x}_{f 0}}\right)
$$

or

$$
g(\ln r, t=0)=3 L_{0}\left(\frac{x}{\bar{x}_{f 0}}\right)^{2} \exp \left(-\frac{x}{\bar{x}_{f 0}}\right),
$$

is assumed. The liquid water content is set to $L_{0}=$ $1 \mathrm{~g} \mathrm{~m}^{-3}$ for most of the discussions and the initial mean mass $\bar{x}_{f 0} \equiv L_{0} / N_{0}=3.3 \times 10^{-12} \mathrm{~kg}$ or the corresponding mean radius $\bar{r}_{f 0} \equiv\left(3 \bar{x}_{f 0} / 4 \pi \rho_{w}\right)^{1 / 3}=9.3 \mu \mathrm{m}$.

The integral form of the kinetic collection equation is solved by a recently developed Bin Integral Method with Gauss Quadrature (BIMGQ; Wang et al. 2007). BIMGQ utilizes an extended linear binwise distribution and the concept of pair interaction to redistribute the mass over new size classes as a result of collision-coalescence. Wang et al. (2007) compared the method with existing numerical approaches for KCE including the method of Berry and Reinhardt (1974), the linear flux method of Bott (1998), and the linear discrete method of Simmel et al. (2002). They showed that BIMGQ has a comparable, or better, accuracy and convergence behavior and is computationally efficient. Here we used a small bin mass ratio of $\alpha=2^{1 / 4}$ to ensure an accurate numerical integration of the KCE by BIMGQ (Wang et al. 2007).

As in some of the previous studies (Berry and Reinhardt 1974; Bott 1998; Simmel et al. 2002), the kinetic collection equation does not explicitly include the transport term due to sedimentation in clouds, but the effect of sedimentation on collection kernel is considered. If the cloud system is assumed to be vertically homogeneous, the inclusion of the sedimentation in the collection kernel may be adequate. For rain initiation stage, the droplet settling velocity is on the order of 10 $\mathrm{cm} \mathrm{s}^{-1}$ and is less than the rms fluctuation velocity of air turbulence, turbulent mixing may maintain a reasonable level of homogeneity.

First, we plot in Figs. 10-12 the mass density distribution of cloud droplets every $10 \mathrm{~min}$ after the initial time on both linear and logarithmic scales. Five curves in each plot represent five different collection kernels. Clearly, the ZWW-RW kernel produces the fastest growth, with the second peak at larger size appearing before $10 \mathrm{~min}$, showing a very early activation of the accretion mode. On the other hand, the mZWWnogr kernel (excluding the gravitational mechanism) results in the slowest growth and only produces a very weak 

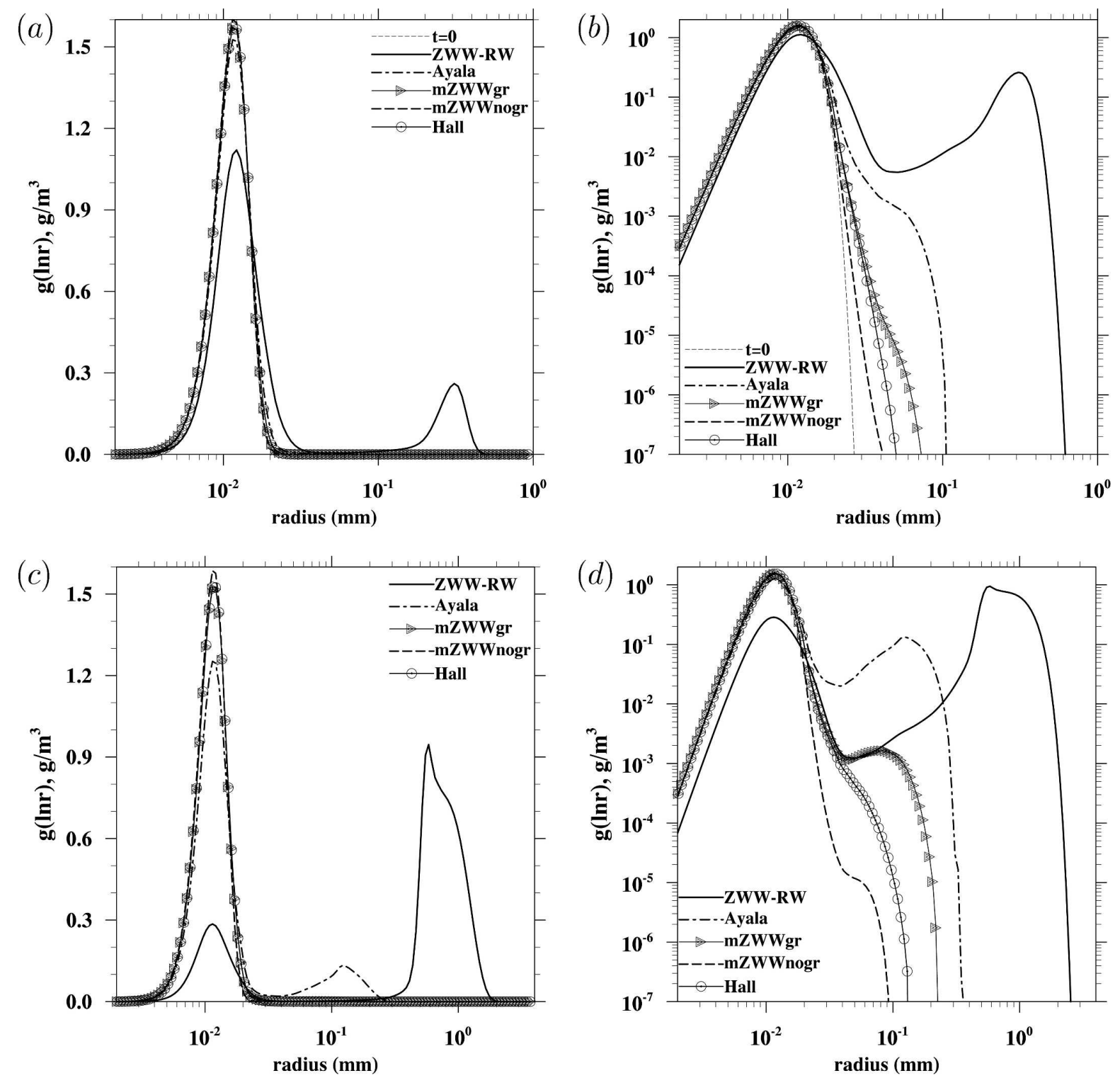

FIG. 10. Mass density distributions at (a) $t=10 \mathrm{~min}$, (b) $t=10 \mathrm{~min}$ on logarithmic scale, (c) $t=20 \mathrm{~min}$, and (d) $t=20 \mathrm{~min}$ on logarithmic scale. The flow dissipation rate and rms velocity fluctuation are $\varepsilon=300 \mathrm{~cm}^{2} \mathrm{~s}^{-3}$ and $u^{\prime}=202 \mathrm{~cm} \mathrm{~s}^{-1}$.

secondary peak at $t=60 \mathrm{~min}$. The other three kernels all generate the secondary peak after $40 \mathrm{~min}$. At $t=$ $30 \mathrm{~min}$, the percentage of mass for droplets larger than $100 \mu \mathrm{m}$ is $93 \%, 76 \%, 60 \%, 0.32 \%$, and $0 \%$ for the ZWW-RW kernel, the Ayala kernel, the mZWWgr kernel, the Hall kernel, and the mZWWnogr kernel, respectively. This shows that air turbulence can speed up the formation of drizzle droplets, but the actual impact depends in a complex manner on the turbulent collision kernel.

As indicated earlier, there are roughly three phases of growth (Berry and Reinhardt 1974): 1) the autoconversion phase in which the self-collections of the small cloud droplets near the peak of the initial size distribution slowly shift the initial peak of the size distribution and, at the same time, transfer mass to larger sizes by a weak accretion mechanism; 2) the accretion phase in which the accretion mode dominates over the autoconversion mode and serves to quickly transfer mass from the initial peak to the newly formed secondary peak at a larger size; and 3) the large hydrometeor selfcollection phase in which the self-collections of large droplets near the second peak now dominate over the accretion mode, as the initial peak is diminishing and the second peak is gaining strength.

We shall now develop a method to identify these 

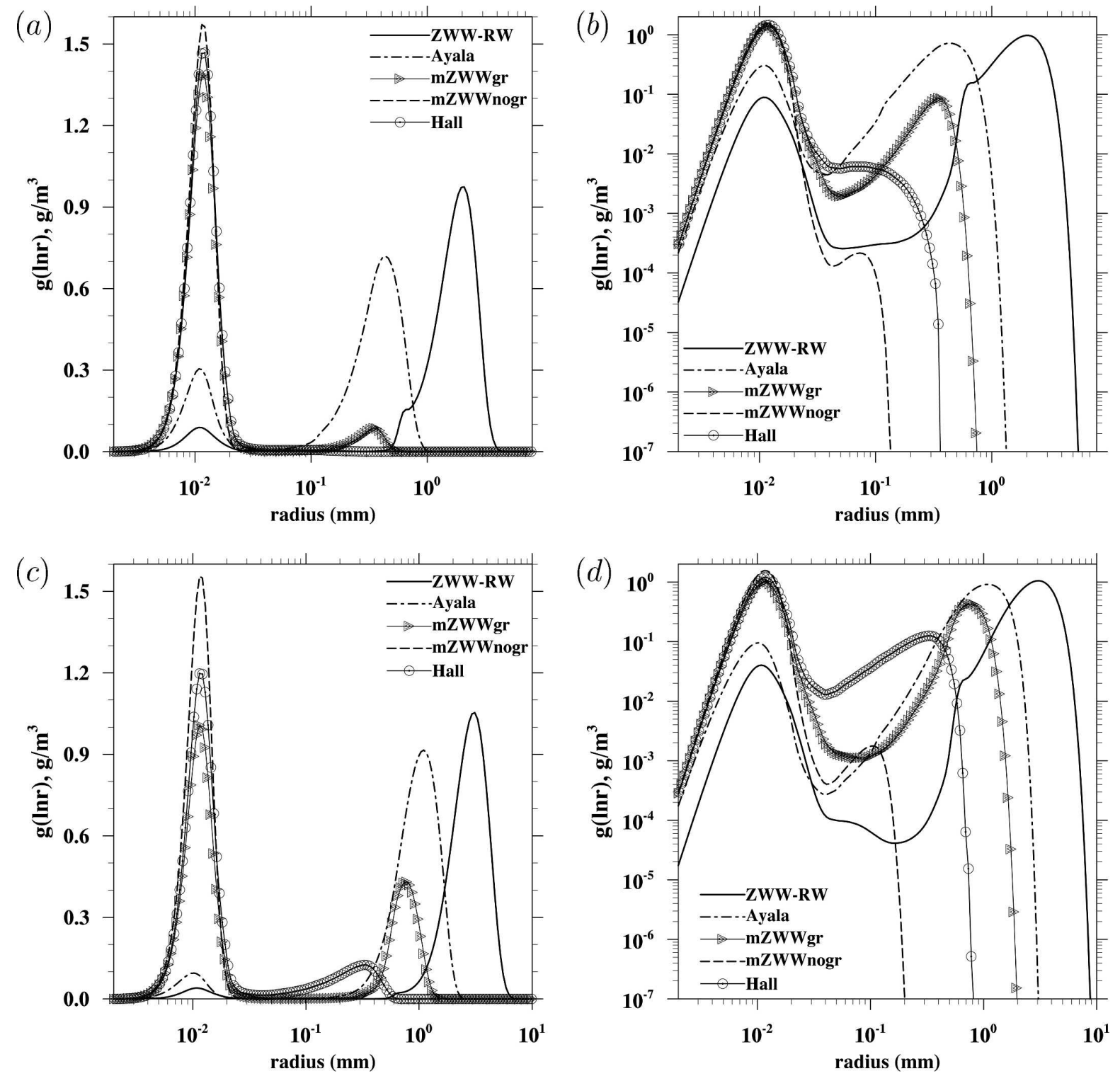

FIG. 11. Mass density distributions at (a) $t=30 \mathrm{~min}$, (b) $t=30 \mathrm{~min}$ on logarithmic scale, (c) $t=40 \mathrm{~min}$, and (d) $t=40 \mathrm{~min}$ on logarithmic scale. The flow dissipation rate and rms velocity fluctuation are $\varepsilon=300 \mathrm{~cm}^{2} \mathrm{~s}^{-3}$ and $u^{\prime}=202 \mathrm{~cm} \mathrm{~s}^{-1}$.

three phases by examining the net rate of transfer of mass density in each bin, $\partial g(\ln r, t) / \partial t$. Figure 13 displays the distribution of $\partial g / \partial t$ every $1 \mathrm{~min}$ for the case of the Ayala kernel. This net rate of transfer was directly solved in the BIMGQ approach. It is noted that $\partial g / \partial t$ can be either positive or negative, with the total integral over the whole size range equal to zero due to the mass conservation. Note that the curves in Fig. 13 are shifted by an amount proportional to time, and the level at the very left should be read as zero for each curve. At any given time, a positive $\partial g / \partial t$ for a given size bin implies that the mass density for that size bin is increasing. The three phases are clearly visible in Fig. 13. During phase
$1, \partial g / \partial t$ is nonzero mainly near the initial peak of the size distribution with a negative region immediately followed by a positive region in $\partial g / \partial t$. Phase 2 is characterized by a largely negative region of $\partial g / \partial t$ near the initial peak and a largely positive region of $\partial g / \partial t$ near the newly formed second peak. There is a size gap in between the two regions during phase 2 , showing that the accretion mode can directly move mass from smaller droplets to droplets much larger in size. Finally, $\partial g / \partial t$ is nonzero mainly near the second peak during the large hydrometeor self-collection phase, again with a negative region immediately followed by a positive region in $\partial g / \partial t$. 

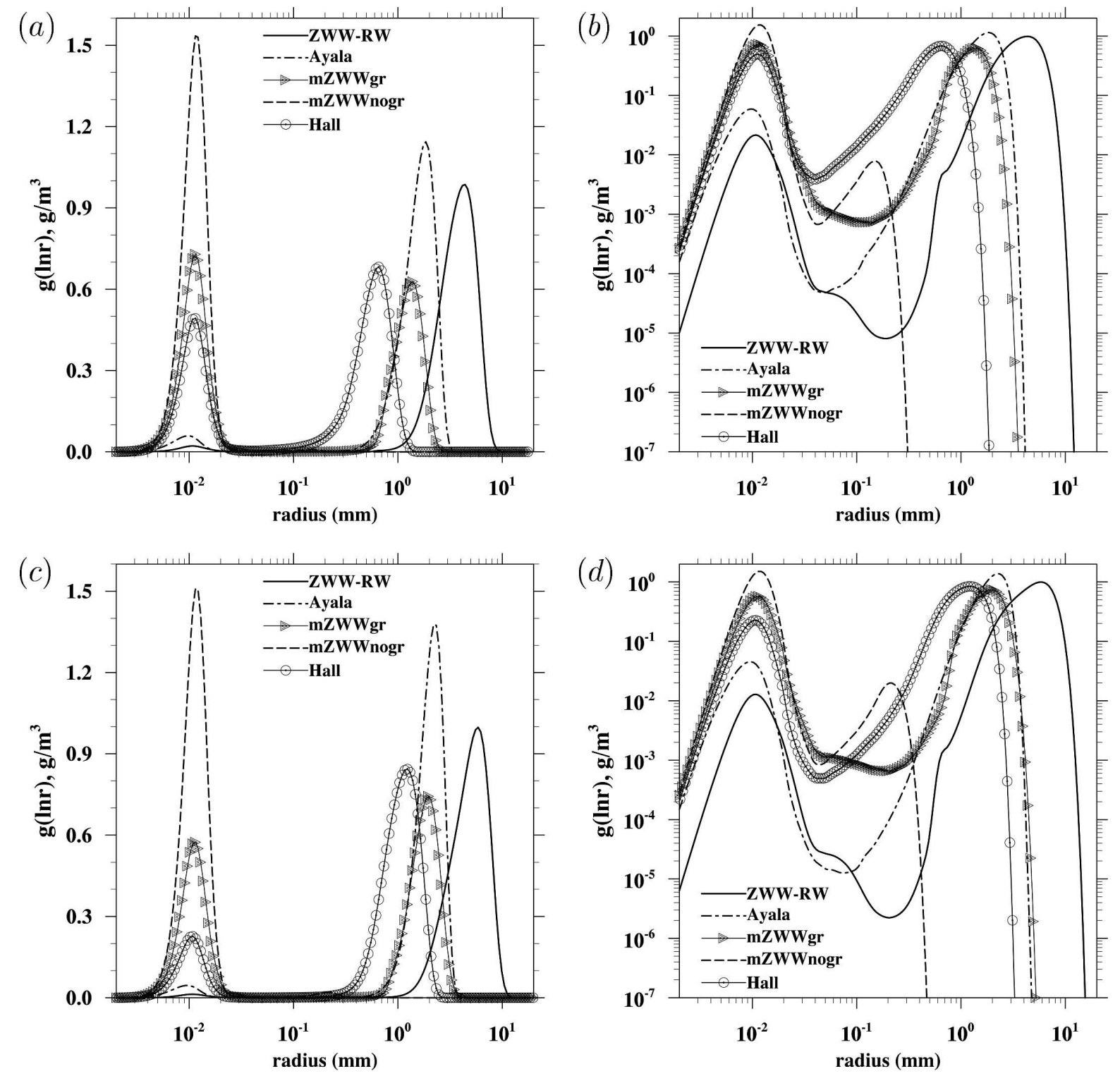

Fig. 12. Mass density distributions at (a) $t=50 \mathrm{~min}$, (b) $t=50 \mathrm{~min}$ on logarithmic scale, (c) $t=60 \mathrm{~min}$, and (d) $t=60 \mathrm{~min}$ on logarithmic scale. The flow dissipation rate and rms velocity fluctuation are $\varepsilon=300 \mathrm{~cm}^{2} \mathrm{~s}^{-3}$ and $u^{\prime}=202 \mathrm{~cm} \mathrm{~s}^{-1}$.

To unambiguously identify the time intervals for the three phases, we plot in Fig. 14, as a function of time, the location in radius, $r_{\max }$, corresponding to the maximum $\partial g / \partial t$ and the location in radius, $r_{\min }$, corresponding to the minimum $\partial g / \partial t$ for the 61 curves shown in Fig. 13. Only times at every 1-min separation are considered. The maximum and minimum values of $\partial g / \partial t$ are also shown in Fig. 14b. For the Ayala kernel, Fig. 14a shows that $r_{\max }$ experiences a sudden jump at about $t=$ $14.5 \mathrm{~min}$. This time marks the end of phase 1 and the beginning of phase 2 . Then at about $t=29.5 \mathrm{~min}, r_{\text {min }}$ shows a sudden increase and this time marks the end of phase 2 and the beginning of phase 3. These transition times correspond well with the detailed $d g(\ln r) / d t$ curves in Fig. 13. Furthermore, while both the maximum value and the minimum value of $\partial g / \partial t$ remain roughly the same for phase 1 , the maximum value of $\partial g / \partial t$ grows rapidly during phase 2 when the accretion mode is switched on. The magnitude of the minimum $\partial g / \partial t$ also grows initially during phase 2 but reaches a peak and then drops during the late part of the accretion phase, as a result of diminishing mass content of small cloud droplets. The magnitudes of the maximum and minimum $\partial g / \partial t$ in general decay during the third phase.

The same procedure was applied to the results based 


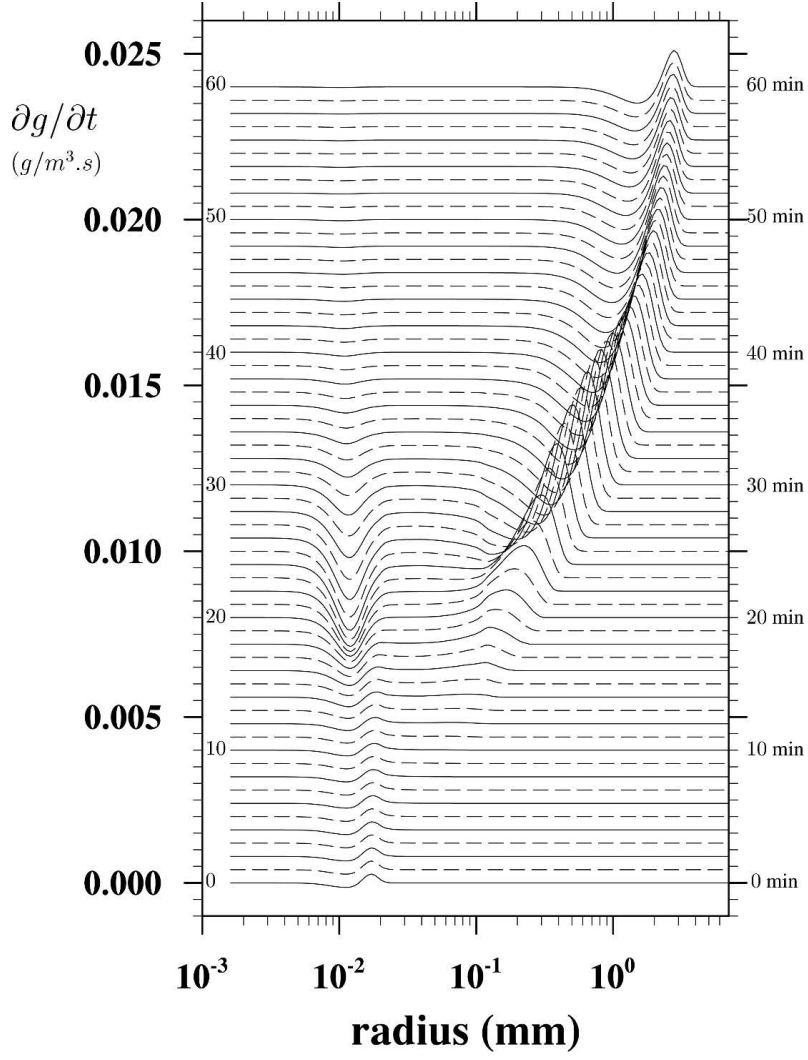

FIG. 13. Distributions of local mass transfer rate for the Ayala kernel at different times with a time increment of $1 \mathrm{~min}$.

on the other four kernels to identify the time interval corresponding to each phase. The results are summarized in Table 2. As far as the generation of drizzle drops, the initiation of the accretion phase is a critical step. The time for the initiation of the accretion phase is about $6.5,14.5,24.5,32.5$, and $51.5 \mathrm{~min}$ for the ZWW-RW kernel, the Ayala kernel, the mZWWgr kernel, the Hall kernel, and the mZWWnogr kernel, respectively. This again shows that air turbulence can significantly reduce the time for the initiation of the accretion phase, and the relative order is consistent with the observed speed of growth shown in Figs. 1012. The transition time from the autoconversion to accretion is closely related to the effectiveness of the autoconversion mechanism in phase 1 . It is important to note that the Ayala kernel does not produce a large enhancement in the collection kernel compared to the ZWW-RW kernel, but it is still quite effective in shortening the time for phase 1 . It follows that the magnitude of the enhancement of the collection kernel is not the most important factor, but the location of the enhancement is the key. If the air turbulence can promote collisions of small droplets, the autoconversion rate is en- (a)

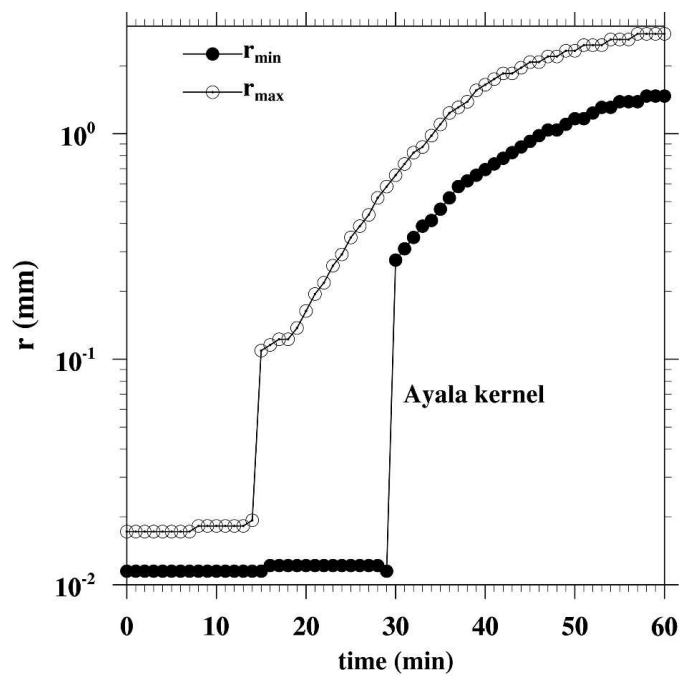

(b)

$\underset{\left(g / m^{3} \cdot s\right)}{\partial g / \partial t}$

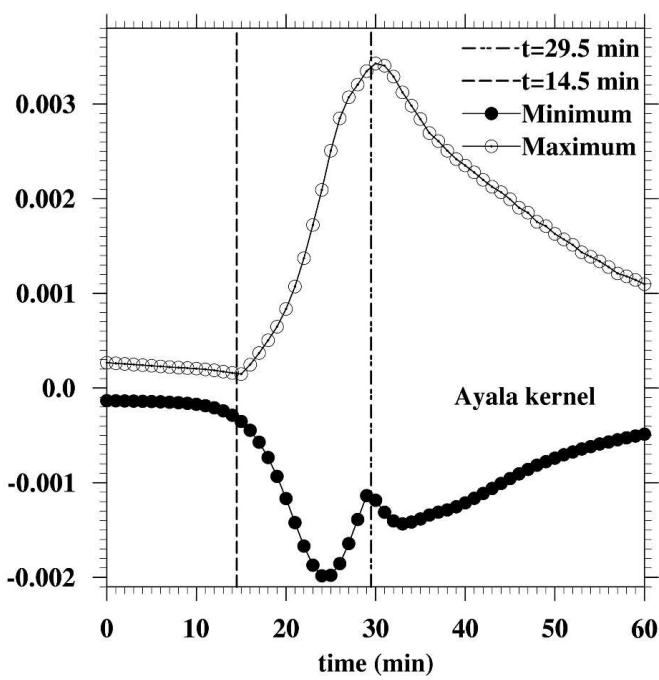

FIG. 14. (a) The droplet radii corresponding to the maximum and minimum $\partial g / \partial t$ as a function of time for the Ayala kernel and (b) the maximum and minimum values of $\partial g / \partial t$ for the Ayala kernel.

hanced so that the accretion phase can be triggered earlier. Once the accretion phase sets in, the gravitational mechanism takes over to continue the growth process. In the absence of the gravitational mechanism such as in the mZWWnogr kernel, the autoconversion phase takes too long to set up the accretion phase, and it makes it almost impossible to grow drizzle drops within a reasonable time.

The time interval for the accretion phase is also reduced by air turbulence, from 19 min for the Hall kernel to 15, 14, and 6 min for the Ayala kernel, the mZWWgr kernel, and the ZWW-RW kernel, respectively. For the case of mWWZa kernel, the accretion phase is not yet completed at $t=60 \mathrm{~min}$. 
TABLE 2. The time interval (min) for each growth phase.

\begin{tabular}{|c|c|c|c|c|c|}
\hline Kernel & Phase 1 & Phase 2 & Phase 3 & {$\left[\frac{\partial g(\ln r, t=0)}{\partial t}\right.$} & ]$_{\max }\left(\mathrm{g} \mathrm{m}^{-3} \mathrm{~s}\right)$ \\
\hline Hall & $t<32.5$ & $32.5<t<51.5$ & $t>51.5$ & 0.138 & $\times 10^{-3}$ \\
\hline Ayala & $t<14.5$ & $14.5<t<29.5$ & $t>29.5$ & $0.267>$ & $\times 10^{-3}$ \\
\hline mZWWnogr & $t<51.5$ & $t>51.5$ & - & 0.0465 & $\times 10^{-3}$ \\
\hline mZWWgr & $t<24.5$ & $24.5<t<38.5$ & $t>38.5$ & 0.146 & $\times 10^{-3}$ \\
\hline ZWW-RW & $t<6.5$ & $6.5<t<12.5$ & $t>12.5$ & 1.160 & $\times 10^{-3}$ \\
\hline
\end{tabular}

Therefore, it turns out that the initiation time for drizzle drops is largely governed by the magnitude of the autoconversion rate during the early part of the time evolution; namely, the consideration of turbulence in collection kernel of small cloud droplets is key to acceleration of rain initiation. Figure $15 \mathrm{~b}$ shows how the time interval $T_{\text {auto }}$ for the autoconversion phase is inversely related to the maximum magnitude of $\partial g / \partial t$ at $t=0$. The initial distributions of $\partial g / \partial t$ are shown in Fig. $15 \mathrm{a}$, and the maximum magnitude of $\partial g / \partial t$ at $t=0$ is listed in Table 2 for each collection kernel. A simple empirical correlation, shown as the straight line in Fig. $15 \mathrm{~b}$, is obtained by fitting the results for the five kernels, as

$$
T_{\text {auto }}=0.07 \times\left[\frac{\partial g(\ln r, t=0)}{\partial t}\right]_{\max }^{-2 / 3} .
$$

Since the initial size distribution contains mostly droplets less than $50 \mu \mathrm{m}$, the maximum magnitude of $\partial g / \partial t$ at $t=0$ is closely related to the initial distribution and the magnitude of the collection kernel for small droplets. The above inverse relation shows that the effect of turbulence on the collection kernel for small droplets (i.e., the AA zone) is likely to be the most important mechanism by which air turbulence can accelerate the growth of cloud droplets. While the accretion phase is typically associated with the formation of a few large cloud droplets, the exact conditions for the transition from autoconversion to accretion is not yet understood on a quantitative level.

Alternative ways of monitoring the growth process are shown in Figs. 16 and 17. First, the radar reflectivity factor, in dBZ (Rogers and Yau 1989), is shown in Fig. 16a for the five kernels. The order in the speed of the growth is shown to be the same as before. Since the radar reflectivity is sixth-order moment in size (or second-order in mass), the rapid growth phase corresponds roughly to the time interval for the accretion phase shown in Table 2. For each collection kernel, two short vertical lines are used to mark the beginning and the end of the accretion phase in Fig. 16. If we choose a radar reflectivity factor of $20 \mathrm{dBZ}$ as the initiation of the drizzle precipitation, as suggested by observations of early radar echoes (Knight et al. 2002), this occurs within the accretion phase for all cases.

Although our model of collision-coalescence does not consider the influence of the changing thermody-

(a)

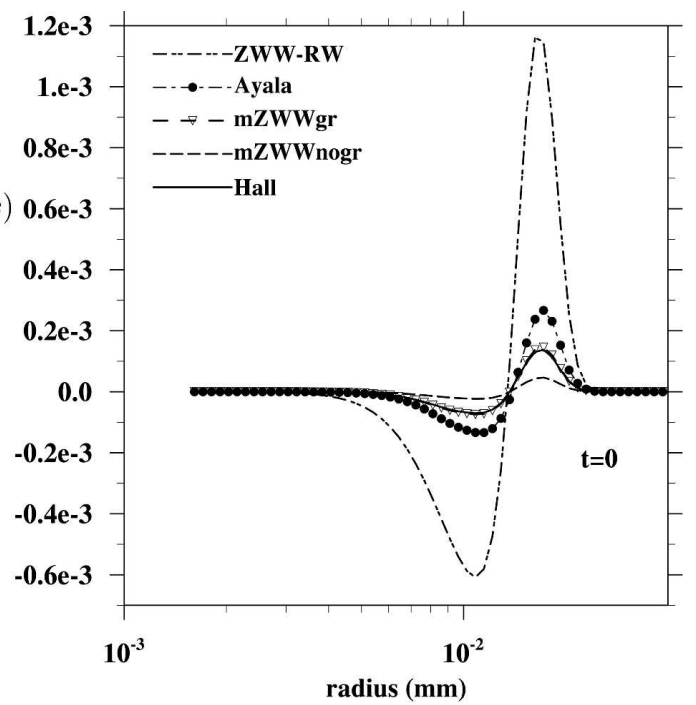

(b)

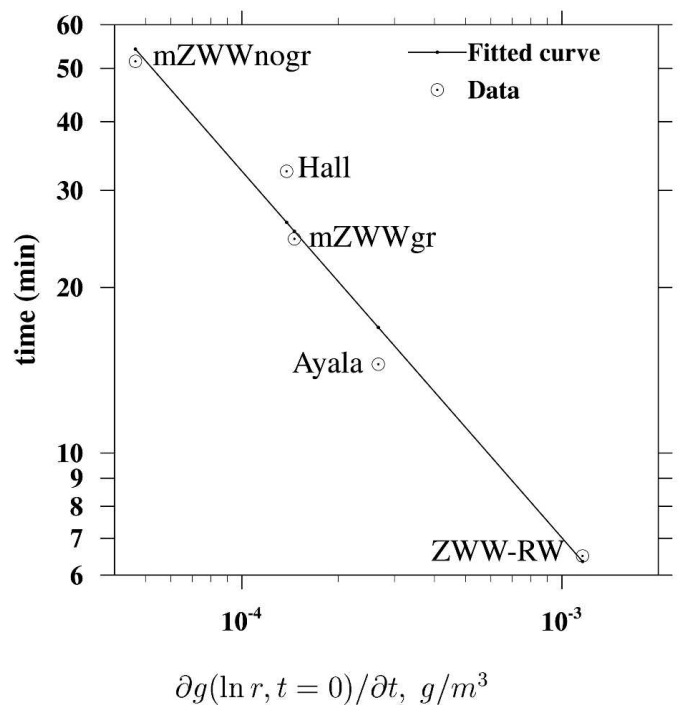

FIG. 15. (a) Distributions of local mass transfer rate at $t=0$ for different collision kernels and (b) the time duration for the autoconversion phase ( $T_{\text {auto }}$ ) as a function of the maximum magnitude of $\partial g / \partial t$ at $t=0$. 

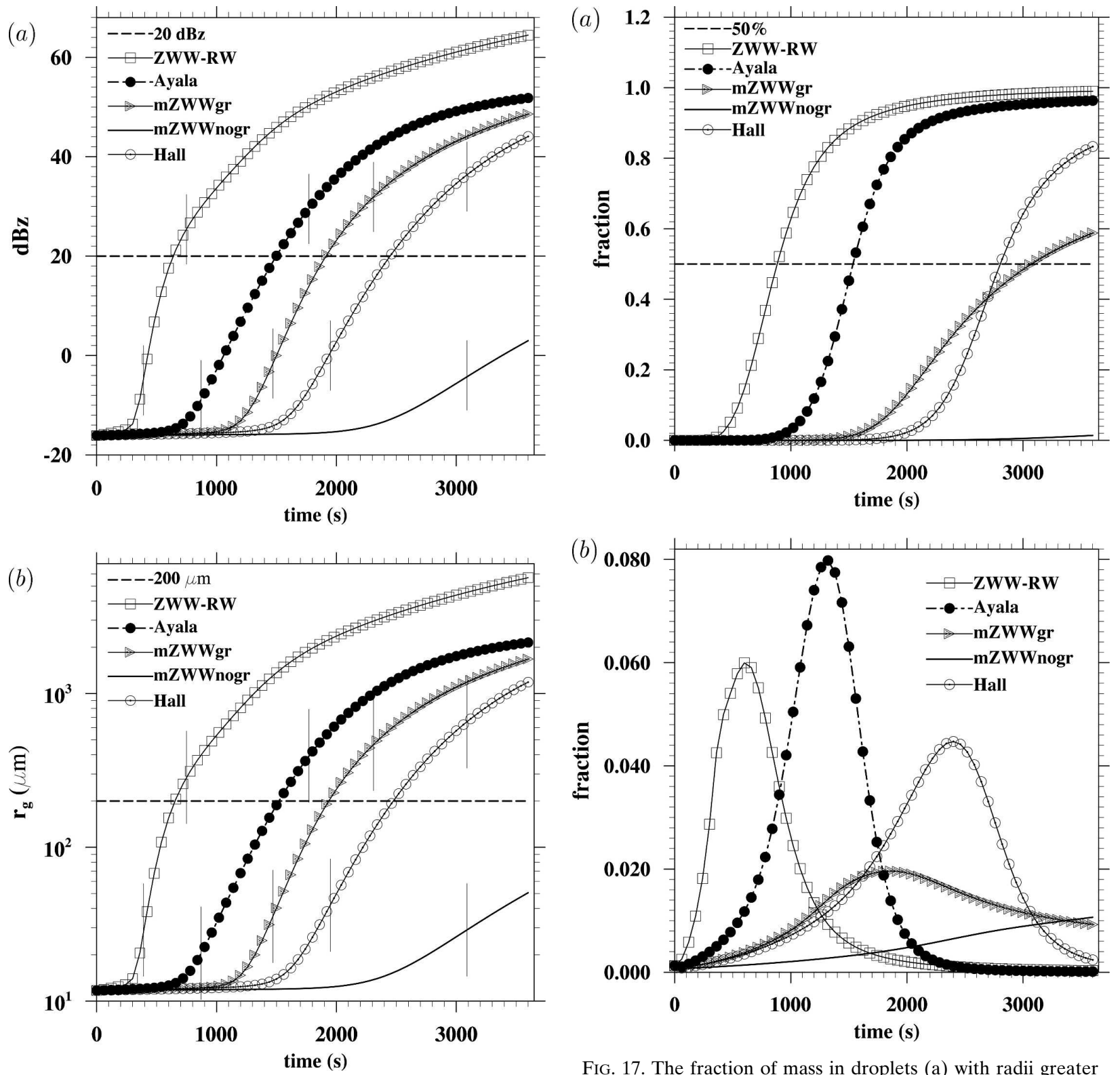

FIG. 16. (a) Radar reflectivity $\mathrm{dB} Z$ as a function of time; (b) mass-weighted mean radius $\left(r_{g}\right)$ as a function of time.

namic and turbulent environments [e.g., within an updraft of a cumulus cloud such as those observed in the Small Cumulus Microphysics Study (Blyth et al. 2003)], we speculate that the rapid growth phase (the accretion phase) may still be compared to observations. The average rate of change in $\mathrm{dB} Z$ during the accretion phase for the Ayala kernel is $2.57 \mathrm{dBZ} \mathrm{min}^{-1}$, comparable to

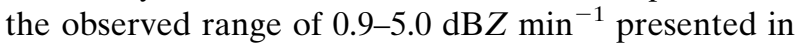
Blyth et al. (2003). This rate is roughly 5.03, 2.40, 1.88, and $0.85 \mathrm{dBZ} \min ^{-1}$ for the ZWW-RW, mWWZb, Hall, and mZWWnogr kernels, respectively. One may

FIG. 17. The fraction of mass in droplets (a) with radii greater than $50 \mu \mathrm{m}$ and (b) with radii from 20 to $100 \mu \mathrm{m}$.

conclude that the ZWW-RW kernel produces a too fast rate of growth, while the mZWWnogr kernel (i.e., without the gravitational mechanism) results in a rate that is too slow. This comparison is not meant to be a rigorous one as the initial conditions between our calculations and the observations were not explicitly matched.

Figure 16b shows the time evolution of the droplet radius $r_{g}$ corresponding to the mean mass based on the mass density distribution, see (11b) of Berry and Reinhardt (1974). Here $r_{g}$ describes approximately the location of the second peak at the larger size. The time evolution of $r_{g}$ is similar to that of radar reflectivity. If 
TABLE 3. Characteristic times (s) for the growth of cloud droplets.

\begin{tabular}{lcccc}
\hline \multicolumn{1}{c}{ Kernel } & $t_{1}$ & $t_{2}$ & $t_{3}$ & $t_{4}$ \\
\hline Hall & 2448 & 2474 & 2804 & 2400 \\
Ayala & 1498 & 1519 & 1536 & 1320 \\
mZWWnogr & - & - & - & - \\
mZWWgr & 1913 & 1935 & 3070 & 1860 \\
ZWW-RW & 640 & 653 & 883 & 600 \\
\hline
\end{tabular}

we choose a value of $r_{g}=200 \mu \mathrm{m}$ as the initiation of the drizzle precipitation, we again conclude that this initiation takes place within the accretion phase for all cases.

Finally, the fraction of mass in the mass distribution for droplets larger than $50 \mu \mathrm{m}$ in radius is shown in Fig. $17 \mathrm{a}$ and the fraction of mass for droplets in the size range from 20 to $100 \mu \mathrm{m}$ is shown in Fig. 17b. The rapid growth phase in Fig. 17a and the occurrence of the peak in Fig. 17b follow the same order for the five different kernels. Specifically, the peaks in Fig. 17b all occur within the accretion phase for each collection kernel.

Four characteristic growth times are extracted from Figs. 16 and 17 and they are listed in Table 3. The first growth time $t_{1}$ marks the instant when the radar reflectivity reaches $20 \mathrm{dBZ}$. The second growth time $t_{2}$ is the time when $r_{g}$ reaches $200 \mu \mathrm{m}$, the third growth time $t_{3}$ corresponds to the time when at least $50 \%$ of the mass is contained by droplets larger than $50 \mu \mathrm{m}$, and the fourth growth time $t_{4}$ represents the time when the fraction of mass in the intermediate size range from 20 to $100 \mu \mathrm{m}$ reaches the maximum. Comparing the data in Table 3 with the data in Table 2, we find that $t_{1}, t_{2}$, and $t_{4}$ all fall within the corresponding time interval for the accretion phase. The value of $t_{3}$ falls either in the late part of the accretion phase or early part of the large hydrometeor self-collection phase.

The above calculations were based on one flow dissipation rate. We repeated the calculation for the four different turbulent kernels for several different flow dissipation rates and three different flow rms fluctuation velocities. [A typical run in Table 4 took less than a minute on a PC Linux workstation with a $2.8-\mathrm{GHz}$ Pentium processor (Wang et al. 2007).] In Table 5, we compare the resulting values of $t_{1}$ and $t_{2}$. Both $t_{1}$ and $t_{2}$ decrease with increasing dissipation rate at a fixed flow fluctuation velocity or with increasing fluctuation velocity at a fixed dissipation rate. These show that the stronger the air turbulence, the shorter the time needed to form drizzle drops. Figure 18 compares $t_{1}$ and $t_{2}$ with our base case, the Hall kernel, which only considers the gravitational mechanism. The same observations can be made. We also list in Table 5 the percentage of reduction in $t_{1}$ and $t_{2}$ relative to the Hall kernel. The air turbulence can shorten the time for the formation of
TABLE 4. Characteristic times (s) for the growth of cloud droplets with the Ayala kernel under different turbulent dissipation rates and rms fluctuation velocities $\left(\mathrm{cm} \mathrm{s}^{-1}\right): L_{0}=$ $1.0 \mathrm{~g} \mathrm{~m}^{-3}$.

\begin{tabular}{cccccc}
\hline $\begin{array}{c}\varepsilon \\
\left(\mathrm{cm}^{2} \mathrm{~s}^{-3}\right)\end{array}$ & $u^{\prime}$ & $t_{1}$ & $t_{2}$ & $\begin{array}{c}\text { Reduction } \\
\text { in } t_{1}(\%)\end{array}$ & $\begin{array}{c}\text { Reduction } \\
\text { in } t_{2}(\%)\end{array}$ \\
\hline \multirow{2}{*}{100} & 100 & 1949 & 1972 & 20.4 & 20.3 \\
& 150 & 1832 & 1855 & 25.2 & 25.0 \\
& 202 & 1738 & 1761 & 29.0 & 28.8 \\
200 & 100 & 1816 & 1837 & 25.8 & 25.7 \\
& 150 & 1685 & 1707 & 31.2 & 31.0 \\
\multirow{3}{*}{300} & 202 & 1584 & 1605 & 35.3 & 35.1 \\
& 100 & 1736 & 1757 & 29.1 & 28.9 \\
& 150 & 1602 & 1623 & 34.6 & 34.4 \\
400 & 202 & 1498 & 1519 & 38.8 & 38.6 \\
& 100 & 1681 & 1702 & 31.3 & 31.2 \\
& 150 & 1547 & 1568 & 36.8 & 36.6 \\
& 202 & 1443 & 1464 & 41.1 & 40.8 \\
\hline
\end{tabular}

drizzle drops roughly from $20 \%$ to $41 \%$ when the dissipation rate varies from 100 to $400 \mathrm{~cm}^{2} \mathrm{~s}^{-3}$ with the range of rms velocity considered.

Finally, in Table 5 we compile a total of 12 runs to investigate different combinations of liquid water content $L_{0}$ and the turbulence dissipation rate $\varepsilon$, when the rms fluctuation $u^{\prime}$ is fixed to $100 \mathrm{~cm} \mathrm{~s}^{-1}$. The initial mean mass radius $\bar{r}_{f 0}$ is always fixed to $9.3 \mu \mathrm{m}$, so increasing $L_{0}$ implies increasing the initial number density $N_{0}$. This is designed to contrast regions of low flow dissipation and high liquid water content (e.g., undiluted cloud core), with regions of high flow dissipation and low liquid water content (cloud edges). The range of $L_{0}$ covers shallow cumulus to deep convective clouds. The range of $\varepsilon$ is taken from the recent helicopter-borne measurements of Siebert et al. (2006). A few interesting observations can be made. The growth times depend very sensitively on the liquid water content,

TABLE 5. Characteristic times (s) for the growth of cloud droplets with the Ayala kernel under different combinations of turbulent dissipation rate $\left(\mathrm{cm}^{2} \mathrm{~s}^{-3}\right)$ and liquid water content $\left(\mathrm{g} \mathrm{m}^{-3}\right)$ : $u^{\prime}=100 \mathrm{~cm} \mathrm{~s}^{-1}$.

\begin{tabular}{rrrccc}
\hline \hline$L_{0}$ & \multicolumn{1}{c}{$\varepsilon$} & \multicolumn{1}{c}{$t_{1}$} & $t_{2}$ & $\begin{array}{c}\text { Reduction } \\
\text { in } t_{1}(\%)\end{array}$ & $\begin{array}{c}\text { Reduction } \\
\text { in } t_{2}(\%)\end{array}$ \\
\hline 1.5 & Hall & 1600 & 1651 & - & - \\
1.5 & 10 & 1478 & 1527 & 7.6 & 7.5 \\
1.5 & 100 & 1271 & 1318 & 20.6 & 20.2 \\
1.5 & 1000 & 996 & 1035 & 37.8 & 37.3 \\
0.3 & Hall & 8702 & 8228 & - & - \\
0.3 & 10 & 8061 & 7606 & 7.4 & 7.6 \\
0.3 & 100 & 6984 & 6556 & 19.7 & 20.3 \\
0.3 & 1000 & 5500 & 5144 & 36.8 & 37.5 \\
3.0 & Hall & 776.4 & 829.2 & - & - \\
3.0 & 10 & 716.4 & 766.4 & 7.7 & 7.6 \\
3.0 & 100 & 614.7 & 662.5 & 20.8 & 20.1 \\
3.0 & 1000 & 479.7 & 519.6 & 38.2 & 37.3 \\
\hline
\end{tabular}




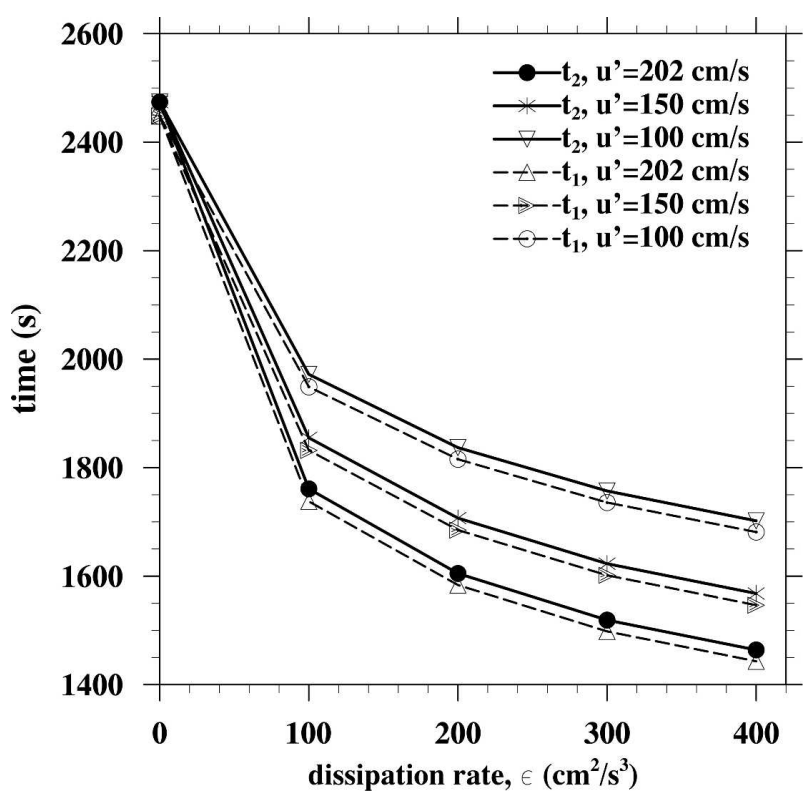

FIG. 18. Characteristic times $t_{1}$ and $t_{2}$ as a function of turbulent dissipation rate for different rms fluctuation velocities.

roughly inversely proportional to the liquid water content for the base case (Hall kernel). This is expected as the kinetic collection equation has a self-similarity: an increase in $N_{0}$ by a factor of $C$ results in exactly a reduction of growth time by a factor of $1 / C$. It follows rigorously that the percentage of reduction in growth time due to air turbulence is independent of the liquid water content. The small differences in Table 5 are merely a reflection of the small numerical integration errors. In all cases including weak turbulence, the effect of turbulence is noticeable. For a given dissipation rate, the relative reduction in growth times are comparable for different liquid water contents. For high liquid water cases, the radar reflectivity reaches the defined threshold first $\left(t_{1}<t_{2}\right)$, while for low liquid water cases the mass-weighted radius reaches the defined threshold first $\left(t_{2}<t_{1}\right)$.

\section{Summary and concluding remarks}

We studied the impact of air turbulence on the growth of cloud droplets using an accurate bin-integral method for the kinetic collection equation and several turbulent collection kernels based on the recent studies by Wang et al. (2000), ZWW01, RW05, and A05. The four turbulent kernels represent different levels of accuracy in treating the effects of air turbulence on geometric collisions through local fluid shear, local fluid acceleration, and preferential concentration. The Ayala kernel is the most realistic turbulent kernel as it is based on direct numerical simulations of turbulent collisions of sedimenting droplets in a turbulent airflow with conditions similar to atmospheric clouds. Unlike other turbulent kernels, the Ayala kernel has several unique characteristics: 1) the overall enhancement by turbulence on geometric collision kernel is very moderate with an average enhancement factor around $2 ; 2$ ) the enhancement covers a broad region in the small droplet size range owing to a coupling mechanism between the relative motion due to differential sedimentation and the relative motion due to air turbulence; and 3) the effects of air turbulence are essentially negligible for droplets larger than $100 \mu \mathrm{m}$ in radii owing to their large terminal velocities. We note that, in a recent study, Pinsky et al. (2006) reported an enhancement factor of less than 2 of geometric collision kernel by turbulence for droplets less than $20 \mu \mathrm{m}$, which seems to be very similar to the level shown in the Ayala kernel (see Fig. 9d).

We compared several time scales for warm rain initiation relative to the hydrodynamical-gravitational kernel of Hall (1980). The most important observation is that the time for the warm rain initiation is largely governed by the timing of the transition from the autoconversion to accretion phase. For example, the time evolution after this transition is very similar for the Ayala kernel, the mZWWgr kernel, and the Hall kernel. If we take the Ayala kernel as the most appropriate kernel for the description of collision-coalescence rate in clouds, then the air turbulence at a typical intensity of turbulence with a dissipation rate of $300 \mathrm{~cm}^{2} \mathrm{~s}^{-3}$ and rms fluctuation velocity of $2 \mathrm{~m} \mathrm{~s}^{-1}$ can shorten the time for the formation of drizzle drops by about $39 \%$ based on both radar reflectivity and mass-weighted mean radius when compared with the Hall kernel. If the gravitational mechanism is completely neglected such as in the mZWWnogr kernel, the turbulence mechanism alone would be too slow to produce drizzles in a reasonable time. The ZWW-RW kernel used in RW05 overestimates the turbulent collision kernel by as much as two orders of magnitude and is shown to lead to an unrealistic reduction (by a factor of 4 ) in rain initiation time by air turbulence.

Another contribution of this study is a method to unambiguously identify the time intervals for the three phases of growth by collision-coalescence as defined qualitatively by Berry and Reinhardt (1974). We used the maximum and minimum of the local mass-density transfer rate to locate the time intervals of the three phases. We found that the air turbulence has the strongest impact on the autoconversion phase, which is typically the longest phase for warm rain initiation. The overall implication is that a moderate increase of collection kernel by air turbulence over the size range of small cloud droplets (less than $50 \mu \mathrm{m}$ in radii) can have 
a significant impact on the warm rain initiation process. This mechanism for growing small cloud droplets is different from the enhanced growth due to the presence of giant and ultragiant nuclei (e.g., Johnson 1982; Blyth et al. 2003). Our mechanism shortens the growth time for the autoconversion phase by moving droplet mass into the size range so that the accretion phase can be activated spontaneously, while the presence of giant and ultragiant nuclei increases the growth by the accretion mode relative to the autoconversion mode on a few lucky giant particles.

We should note that the speedup in the formation of drizzles observed in this study is somewhat less than what is needed to fully close the gap between previous calculations and observations. The parameter space studied is limited as far as liquid water content and the level of turbulence intensity are considered. There are also simplifications such as no fallout of droplets and the assumed single initial spectrum width. Condensation was also not explicitly included so the cloud condensation nuclei $(\mathrm{CCN})$ input and other related variations are not considered. These limitations and simplifications will need to be addressed in future studies.

Although this study does not include the effect of air turbulence on the collision efficiency (Wang et al. 2005a), the observations made here are very relevant to the impact of the enhanced collision efficiency by air turbulence on the warm rain initiation as the enhanced collision efficiency occurs also in the small size range with the droplet radius less than $50 \mu \mathrm{m}$. For droplets less than $50 \mu \mathrm{m}$, Wang et al. (2005a) showed that the enhancement of collision efficiency by turbulence is at least comparable to the enhancement factor on geometric collision rate as shown here in the Ayala kernel. Therefore, should the enhancement of collision efficiency by turbulence be included, the airflow turbulence could likely shorten the time for the formation of drizzle drops even further. This could then resolve the discrepancy between the observed time for rain initiation and the predicted time based on the gravitational mechanism alone. At this stage, further research is needed to quantify the effects of air turbulence on collision rate and collision efficiency before a complete collision kernel can be developed.

Acknowledgments. This study has been supported by the National Science Foundation through Grants ATM-0114100 and ATM-0527140, and by the National Center for Atmospheric Research (NCAR). The support of NCAR Faculty Fellowship to LPW is gratefully acknowledged. We are also grateful to Dr. Nicole Riemer for her assistance in reproducing the results in Riemer and Wexler (2005).

\section{APPENDIX A}

\section{List of Symbols}

$\mathrm{dBZ} \quad$ Radar reflectivity factor in the form of 10 $\log _{10} Z$

$d t \quad$ Time step, in $\mathrm{s}$

$E_{i j}^{t} \quad$ Collision efficiency for the turbulent mechanism

$E_{i j}^{s} \quad$ Collision efficiency for the gravitational mechanism

$g_{i j} \quad$ Radial distribution function for droplet pairs of radii $r_{i}$ and $r_{j}$

Gravitational acceleration vector, $|\mathbf{g}|=980$ $\mathrm{cm} \mathrm{s}^{-2}$

g

$g(\ln r) \quad$ Mass density function, in $\mathrm{g} \mathrm{m}^{-3}$

$K_{i, j} \quad$ Collection kernel, in $\mathrm{cm}^{3} \mathrm{~s}^{-1}$

$L_{e} \quad$ Eulerian integral length scale of turbulence, in $\mathrm{cm}$

$L \quad$ Liquid water content, in $\mathrm{g} \mathrm{m}^{-3}$

$n(x, t) \quad$ Number density distribution

$N \quad$ Number density, in $\mathrm{cm}^{-3}$

$R \quad$ Geometric collision radius $\left(R=r_{i}+r_{j}\right)$, in $\mathrm{cm}$ or $\mu \mathrm{m}$

$R_{\lambda} \quad \equiv u^{\prime} \lambda / \nu=u^{\prime 2} \sqrt{15 /(\varepsilon \nu)}$, Taylor-microscale Reynolds number

$\mathrm{Re}_{p} \quad$ Droplet Reynolds number

$r \quad$ Droplet radius, in $\mathrm{cm}$ or $\mu \mathrm{m}$

$\bar{r}_{f 0} \quad$ Initial mean-mass radius, in $\mathrm{cm}$ or $\mu \mathrm{m}$

St Droplet Stokes number

$T_{\text {auto }} \quad$ Time interval for the autoconversion phase, in $\mathrm{s}$

$T_{e} \quad$ Large-eddy turnover time of turbulence, in $\mathrm{s}$

$T_{L} \quad$ Lagrangian integral time of turbulence, in $\mathrm{s}$

$t \quad$ Time, in $\mathrm{s}$

$u^{\prime} \quad$ Flow rms fluctuation velocity in a given direction, in $\mathrm{cm} \mathrm{s}^{-1}$

$v_{n} \quad$ Droplet terminal velocity based on nonlinear drag, in $\mathrm{cm} \mathrm{s}^{-1}$

$v_{k} \quad \equiv(\nu \varepsilon)^{1 / 4}$, Kolmogorov velocity scale, in $\mathrm{cm} \mathrm{s}^{-1}$

$v_{\mathrm{s}} \equiv \tau_{s}|\mathbf{g}|$, Stokes terminal velocity, in $\mathrm{cm} \mathrm{s}^{-1}$

$\left\langle\left(v^{(i)} v^{\prime(j)}\right)\right\rangle$ Particle velocity cross-covariance, in $\mathrm{cm}^{2} \mathrm{~s}^{-2}$

$\left\langle\left(v^{\prime(k)}\right)^{2}\right\rangle \quad$ Mean-square particle velocity, in $\mathrm{cm}^{2} / \mathrm{s}^{2}$

$w_{r} \quad$ Droplet radial relative velocity, in $\mathrm{cm} / \mathrm{s}$

$\left\langle w_{r, \text { accel }}^{2}\right\rangle \quad$ Contribution to the radial relative velocity due to differential fluid acceleration, in $\mathrm{cm} \mathrm{s}^{-1}$

$\left\langle w_{r, \text { shear }}^{2}\right\rangle \quad$ Contribution to the radial relative velocity due to local fluid shear, in $\mathrm{cm} \mathrm{s}^{-1}$

Droplet mass, in $\mathrm{kg}$ or $\mathrm{g}$

Initial mean mass, in $\mathrm{kg}$ or $\mathrm{g}$

Radar reflectivity factor, in $\mathrm{mm}^{6} \mathrm{~m}^{-3}$ 


\section{Greek Symbols}

$\eta$

$\mu$

$\nu$

$\lambda$

$\phi$

$\rho_{a}$

$\rho_{w}$

$\rho_{i j}$

$\tau_{k}$

$\tau_{p}$

$\tau_{s}$

$\tau_{n}$

$\tau_{T}$

$\theta_{i}$ pair
Turbulent dissipation rate, in $\mathrm{cm}^{2} \mathrm{~s}^{-3}$ $\equiv\left(\nu^{3} / \varepsilon\right)^{1 / 4}$, Kolmogorov length scale, in $\mathrm{cm}$ Dynamic viscosity of the air $=1.818 \times 10^{-4}$ $\mathrm{g} \mathrm{cm}^{-1} \mathrm{~s}^{-1}$

$=\mu / \rho_{a}$, kinematic air viscosity, in $\mathrm{cm}^{2} \mathrm{~s}^{-1}$

Taylor microscale, in $\mathrm{cm}$

Ratio of inertial response times of a droplet

Air density $=0.001225 \mathrm{~g} \mathrm{~cm}^{-3}$

Water density $=1 \mathrm{~g} \mathrm{~cm}^{-3}$

Pair distribution correlation coefficient

Variance of the radial relative velocity due to turbulence, in $\mathrm{cm}^{2} \mathrm{~s}^{-2}$

$\equiv \sqrt{\nu / \varepsilon}$, Kolmogorov time scale, in $\mathrm{s}$

Inertial response time of a droplet, $s$

$\equiv 2 \rho_{w} r_{i}^{2} /\left(9 \nu \rho_{a}\right)$, inertial response time for Stokes drag, in $\mathrm{s}$

Inertial response time based on nonlinear drag, defined in Eq. (5)

Lagrangian Taylor microscale time, in $\mathrm{s}$ $=2.5 \tau_{p_{i}} / T_{L}$

\section{APPENDIX B}

\section{Formulation of the ZWW-RW Kernel}

Here we present the detail parameterization of the ZWW01 kernel for nonsettling particles. As indicated in Eqs. (1)-(3), four kinematic elements need to be modeled: the relative velocity $\left\langle w_{r \text {,accel }}^{2}\right\rangle$ due to differential fluid acceleration, the relative velocity $\left\langle w_{r, \text { shear }}^{2}\right\rangle$ due to local fluid shear, the radial distribution functions $\left[g_{i i}(r=R)\right.$ and $\left.g_{j j}(r=R)\right]$ for monodisperse particles, and the cross-correlation coefficient $\rho_{i j}$.

The shear term is easily modeled as (Saffman and Turner 1956; Wang et al. 1998b)

$$
\left\langle w_{r, \text { shear }}^{2}\right\rangle=\frac{1}{15} v_{k}^{2}\left(\frac{R}{\eta}\right)^{2},
$$

where $\eta$ is the turbulence Kolmogorov length and $v_{k}$ is the Kolmogorov velocity. A complete list of symbols and their interrelationships are provided in appendix A. The acceleration term is given as

$$
\left\langle w_{r, \text { accel }}^{2}\right\rangle=C_{w}(z)\left\langle w_{\text {accel }, 0}^{2}\right\rangle,
$$

with

$$
C_{w}(z)=1.0+0.6 \exp \left[-(z-1)^{1.5}\right] .
$$

Here $z=\max \left(\theta_{i} / \theta_{j}, \theta_{j} / \theta_{i}\right), \theta_{i}=2.5 \tau_{s i} / T_{L}$, where $\tau_{s i}$ is the cloud droplet Stokes inertial response time. The flow Lagrangian integral time $T_{L}$ is estimated as $T_{L} \approx u^{\prime 2} / \varepsilon$, where $u^{\prime}$ is the turbulent rms fluctuation velocity and $\varepsilon$ is the flow viscous dissipation rate. Since the droplet density is much larger than the air density, the expression for $\left\langle w_{r, \text { accel }}^{2}\right\rangle$ taken from Kruis and Kusters (1997) becomes

$$
\left\langle w_{\text {accel }, 0}^{2}\right\rangle=\frac{u^{\prime 2} \gamma}{\gamma-1}\left\{\left(\theta_{i}+\theta_{j}\right)-\frac{4 \theta_{i} \theta_{j}}{\theta_{i}+\theta_{j}}\left[\frac{1+\theta_{i}+\theta_{j}}{\left(1+\theta_{i}\right)\left(1+\theta_{j}\right)}\right]^{1 / 2}\right\}\left[\frac{1}{\left(1+\theta_{i}\right)\left(1+\theta_{j}\right)}-\frac{1}{\left(1+\gamma \theta_{i}\right)\left(1+\gamma \theta_{j}\right)}\right],
$$

where $\gamma$ is a function of $z$ :

$$
\gamma=z \times 0.183 \frac{u^{\prime 2}}{(\varepsilon \nu)^{1 / 2}} .
$$

The monodisperse radial distribution function $g_{k k}(R)$ ( $k=i$ or $j$ ) is parameterized as

$$
\begin{aligned}
g_{k k}(R)= & 1+y_{0}(\mathrm{St})\left[1-z_{0}^{2}(\mathrm{St})\right] \\
& +z_{0}^{2}(\mathrm{St}) R_{\lambda}\left\{y_{1}(\mathrm{St})\left[1-z_{1}(\mathrm{St})\right]\right. \\
& \left.+y_{2}(\mathrm{St}) z_{1}(\mathrm{St})+y_{3}(\mathrm{St}) z_{2}(\mathrm{St})\right\}
\end{aligned}
$$

where $R_{\lambda}$ is the Taylor microscale Reynolds number, the Stokes number St is defined as $\mathrm{St} \equiv \tau_{s i} / \tau_{k}$, and $\tau_{k}$ is the Kolmogorov time scale. The functions $y_{i}(\mathrm{St})$ and $z_{i}(\mathrm{St})$ are expressed as

$$
\begin{aligned}
& y_{0}(\mathrm{St})=18 \mathrm{St}^{2}, \quad y_{1}(\mathrm{St})=0.36 \mathrm{St}^{2.5} \exp \left(-\mathrm{St}^{2.5}\right), \\
& y_{2}(\mathrm{St})=0.24 \exp (-0.5 \mathrm{St}) \\
& y_{3}(\mathrm{St})=0.013 \exp (-0.07 \mathrm{St})
\end{aligned}
$$

and

$$
z_{0}(\mathrm{St})=\frac{1}{2}\left(1+\tanh \frac{\mathrm{St}-0.5}{0.25}\right), \quad z_{1}(\mathrm{St})=\frac{1}{2}\left(1+\tanh \frac{\mathrm{St}-1.25}{0.1}\right), \quad z_{2}(\mathrm{St})=\frac{1}{2}\left(1+\tanh \frac{\mathrm{St}-6.5}{2.5}\right) .
$$

The correlation coefficient $\rho_{i j}(\phi)$ is described as

$$
\rho_{i j}(\phi)=2.6 \exp (-\phi)+0.205 \exp (-0.0206 \phi) \times \frac{1}{2}[1.0+\tanh (\phi-3)]
$$

where $\phi \equiv \tau_{s j} / \tau_{s i}$. 


\section{APPENDIX C}

\section{Formulation of the Ayala Kernel}

In this appendix, we fill in the expressions needed to complete the Ayala kernel as outlined by Eqs. (7)-(10).
The detailed derivations of the following expressions can be found in A05. First, the velocity variance and cross variance for the motion of droplets due to turbulence are described as

$$
\left\langle\left(v^{\prime(k)}\right)^{2}\right\rangle=\frac{u^{\prime 2}}{\tau_{p k}}\left[b_{1} d_{1} \Psi\left(c_{1}, e_{1}\right)-b_{1} d_{2} \Psi\left(c_{1}, e_{2}\right)-b_{2} d_{1} \Psi\left(c_{2}, e_{1}\right)+b_{2} d_{2} \Psi\left(c_{2}, e_{2}\right)\right], \quad k=i \text { or } j
$$

and

$$
\left\langle\left({v^{\prime(i)}}^{\left.\boldsymbol{v}^{(j)}\right)}\right)\right\rangle=\frac{u^{\prime 2} f(R)}{\tau_{p i} \tau_{p j}}\left[b_{1} d_{1} \Phi\left(c_{1}, e_{1}\right)-b_{1} d_{2} \Phi\left(c_{1}, e_{2}\right)-b_{2} d_{1} \Phi\left(c_{2}, e_{1}\right)+b_{2} d_{2} \Phi\left(c_{2}, e_{2}\right)\right]
$$

where

$$
\begin{aligned}
f(R)= & \frac{1}{2\left(1-2 \chi^{2}\right)^{1 / 2}} \times\left\{\left(1+\sqrt{1-2 \chi^{2}}\right) \exp \left[-\frac{2 R}{\left(1+\sqrt{1-2 \chi^{2}}\right) L_{e}}\right]\right. \\
& \left.-\left(1-\sqrt{1-2 \chi^{2}}\right) \exp \left[-\frac{2 R}{\left(1-\sqrt{1-2 \chi^{2}}\right) L_{e}}\right]\right\} .
\end{aligned}
$$

The parameters $b_{1}, b_{2}, c_{1}, c_{2}, d_{1}, d_{2}, e_{1}$, and $e_{2}$ are defined as

$$
\begin{aligned}
& b_{1}=\frac{1+\sqrt{1-2 z^{2}}}{2 \sqrt{1-2 z^{2}}}, \quad b_{2}=\frac{1-\sqrt{1-2 z^{2}}}{2 \sqrt{1-2 z^{2}}}, \quad c_{1}=\frac{\left(1+\sqrt{1-2 z^{2}}\right) T_{L}}{2}, \quad c_{2}=\frac{\left(1-\sqrt{1-2 z^{2}}\right) T_{L}}{2}, \\
& d_{1}=\frac{1+\sqrt{1-2 \chi^{2}}}{2 \sqrt{1-2 \chi^{2}}}, \quad d_{2}=\frac{1-\sqrt{1-2 \chi^{2}}}{2 \sqrt{1-2 \chi^{2}}}, \quad e_{1}=\frac{\left(1+\sqrt{1-2 \chi^{2}}\right) L_{e}}{2}, \quad e_{2}=\frac{\left(1-\sqrt{1-2 \chi^{2}}\right) L_{e}}{2},
\end{aligned}
$$

respectively. Here, $z \equiv \tau_{k} / T_{L}$ and $\chi \equiv \sqrt{2} \lambda / L_{e}$. The The function $\Phi(\xi, \zeta)$ appeared in the cross-variance Eulerian integral length $L_{e}$ is modeled as $L_{e}=0.5 u^{\prime 3} / \varepsilon$. equation is given as

$$
\begin{aligned}
\Phi(\xi, \zeta)= & {\left[1 /\left(\frac{v_{p_{j}}}{\zeta}-\frac{1}{\tau_{p_{j}}}-\frac{1}{\xi}\right)-1 /\left(\frac{v_{p_{i}}}{\zeta}+\frac{1}{\tau_{p_{i}}}+\frac{1}{\xi}\right)\right]\left(v_{p_{i}}-v_{p_{j}}\right) / 2 \zeta\left(\frac{v_{p_{i}}-v_{p_{j}}}{\zeta}+\frac{1}{\tau_{p_{i}}}+\frac{1}{\tau_{p_{j}}}\right)^{2} } \\
& +\left[\frac{1}{\left(\frac{v_{p_{j}}}{\zeta}\right)^{2}-\left(\frac{1}{\tau_{p_{i}}}+\frac{1}{\xi}\right)^{2}}-\frac{1}{\left(\frac{v_{p_{j}}}{\zeta}+\frac{1}{\tau_{p_{j}}}+\frac{1}{\xi}\right)^{2}}-\frac{v_{p_{j}}}{\left(\frac{v_{p_{j}}}{\zeta}-\frac{1}{\tau_{p_{j}}}-\frac{1}{\xi}\right)^{2}}\right] \times \frac{1}{2 \zeta\left[\frac{1}{\tau_{p_{i}}}-\frac{1}{\xi}+\left(\frac{1}{\tau_{p_{j}}}+\frac{1}{\xi}\right) \frac{v_{p_{i}}}{v_{p_{j}}}\right]} \\
& +\left[\zeta /\left(\frac{v_{p_{i}}}{\zeta}+\frac{1}{\tau_{p_{i}}}+\frac{1}{\xi}\right)-2 \zeta /\left(\frac{v_{p_{j}}}{\zeta}-\frac{1}{\tau_{p_{j}}}-\frac{1}{\xi}\right)-v_{p_{i}} /\left(\frac{v_{p_{i}}}{\zeta}+\frac{1}{\tau_{p_{i}}}+\frac{1}{\xi}\right)^{2}+v_{p_{j}} /\left(\frac{v_{p_{j}}}{\zeta}-\frac{1}{\tau_{p_{j}}}-\frac{1}{\xi}\right)^{2}\right] \\
& \times \frac{1}{2 \zeta\left(\frac{v_{p_{i}}-v_{p_{j}}}{\zeta}+\frac{1}{\tau_{p_{i}}}+\frac{1}{\tau_{p_{j}}}\right)},
\end{aligned}
$$

and the function $\Psi(\xi, \zeta)$ in the variance equation is 


$$
\Psi(\xi, \zeta)=1 /\left(\frac{1}{\tau_{p_{k}}}+\frac{1}{\xi}+\frac{v_{p_{k}}}{\zeta}\right)-v_{p_{k}} /\left[2 \zeta\left(\frac{1}{\tau_{p_{k}}}+\frac{1}{\xi}-\frac{v_{p_{k}}}{\zeta}\right)^{2}\right]
$$

where the particle response time and terminal velocity are based on the nonlinear drag. In Eq. (C3), it is assumed that $v_{n i}>v_{n j}$.

For the radial distribution function described in Eq. (10), the exponent $C_{1}$ and the transition distance $r_{c}$ are both obtained by curve fitting to DNS data. The empirical expression for $C_{1}$ is

$$
C_{1}=\frac{y(S T)}{\left(|\mathbf{g}| /\left(v_{k} / \tau_{k}\right)\right)^{f\left(R_{\lambda}\right)}}
$$

where

$$
\begin{aligned}
y(\mathrm{ST})= & -0.1988 \mathrm{ST}^{4}+1.5275 \mathrm{ST}^{3}-4.2942 \mathrm{ST}^{2} \\
& +5.3406 \mathrm{ST}, \\
f\left(R_{\lambda}\right)= & 0.1886 \exp \left(\frac{20.306}{R_{\lambda}}\right),
\end{aligned}
$$

and $\mathrm{ST} \equiv \max \left(\mathrm{St}_{2}, \mathrm{St}_{1}\right)$. The transition distance $r_{c}$ is expressed as

$$
\left(\frac{r_{c}}{\eta}\right)^{2}=\left|\mathrm{St}_{2}-\mathrm{St}_{1}\right| F\left(a_{o_{g}}, R_{\lambda}\right),
$$

where $a_{o_{g}}$ is

$$
a_{o_{g}}=\frac{11+7 R_{\lambda}}{205+R_{\lambda}}+\frac{\pi}{8}\left(\frac{|\mathbf{g}|}{v_{k} / \tau_{k}}\right)^{2}
$$

and

$$
F\left(a_{o_{g}}, R_{\lambda}\right)=20.115\left(\frac{a_{o_{g}}}{R_{\lambda}}\right)^{1 / 2} .
$$

\section{REFERENCES}

Arenberg, D., 1939: Turbulence as a major factor in the growth of cloud droplets. Bull. Amer. Meteor. Soc., 20, 444-445.

Ayala, O., 2005: Effects of turbulence on the collision rate of cloud droplets. Ph.D. dissertation, University of Delaware, $236 \mathrm{pp}$.

- L.-P. Wang, and W. W. Grabowski, 2007: A hybrid approach for simulating turbulent collisions of hydrodynamically-interacting particles. J. Comput. Phys., 225, 51-73.

Beard, K. V., and H. T. Ochs III, 1993: Warm-rain initiation: An overview of microphysical mechanisms. J. Appl. Meteor., 32, 608-625.

Berry, E. X., and R. I. Reinhardt, 1974: An analysis of cloud drop growth by collection: Part I. Double distributions. J. Atmos. Sci., 31, 1814-1824.

Blyth, A. M., S. G. Lasher-Trapp, W. A. Cooper, C. A. Knight, and J. Latham, 2003: The role of giant and ultragiant nuclei in the formation of early radar echoes in warm cumulus clouds. J. Atmos. Sci., 60, 2557-2572.
Bott, A., 1998: A flux method for the numerical solution of the stochastic collection equation. J. Atmos. Sci., 55, 2284-2293.

Brenguier, J.-L., and W. W. Grabowski, 1993: Cumulus entrainment and cloud droplet spectra: A numerical model within a two-dimensional dynamical framework. J. Atmos. Sci., 50, 120-136.

- T. Bourrianne, A. Coelho, J. Isbert, R. Peytavi, D. Trevarin, and P. Weschler, 1998: Improvements of the droplet size distribution measurements with the Fast-FSSP (Forward Scattering Spectrometer Probe). J. Atmos. Oceanic Technol., 15, 1077-1090.

Chaumat, L., and J.-L. Brenguier, 1998: Droplet spectra broadening and concentration inhomogeneities. Preprints, Conf. on Cloud Physics, Everett, WA, Amer. Meteor. Soc., 514517.

Chun, J., D. L. Koch, S. L. Rani, A. Ahluwalia, and L. R. Collins, 2005: Clustering of aerosol particles in isotropic turbulence. $J$. Fluid Mech., 536, 219-251.

Clift, R., J. R. Grace, and M. E. Weber, 1978: Bubbles, Drops, and Particles. Academic Press, 380 pp.

Dávila, J., and J. C. R. Hunt, 2001: Settling of small particles near vortices and in turbulence. J. Fluid Mech., 440, 117-145.

de Almeida, F. C., 1976: The collisional problem of cloud droplets moving in a turbulent environment-Part I: A method of solution. J. Atmos. Sci., 33, 1571-1578.

_ 1979: The collisional problem of cloud droplets moving in a turbulent environment-Part II: Turbulent collision efficiencies. J. Atmos. Sci., 36, 1564-1576.

Dodin, Z., and T. Elperin, 2002: On the collision rate of particles in turbulent flow with gravity. Phys. Fluids, 14, 2921-2924.

East, T. W. R., and J. S. Marshall, 1954: Turbulence in clouds as a factor in precipitation. Quart. J. Roy. Meteor. Soc., 80, 26-47.

Falkovich, G., A. Fouxon, and M. G. Stepanov, 2002: Acceleration of rain initiation by cloud turbulence. Nature, 419, 151154.

Franklin, C. N., P. A. Vaillancourt, M. K. Yau, and P. Bartello, 2005: Collision rates of cloud droplets in turbulent flow. $J$. Atmos. Sci., 62, 2451-2466.

Furumoto, J., K. Kurimoto, and T. Tsuda, 2003: Continuous observations of humidity profiles with the MU radar-RASS combined with GPS and radiosonde measurements. J. Atmos. Oceanic Technol., 20, 23-41.

Gabilly, A., 1949: On the role that turbulence can play in the coalescence of cloud droplets. Ann. Geophys., 5, 232-234.

Ghosh, S., and P. R. Jonas, 2001: Some analytical calculations on the effect of turbulence on the settling and growth of cloud droplets. Geophys. Res. Lett., 28, 3883-3886.

- J. Dávila, J. C. R. Hunt, A. Srdic, H. J. S. Fernando, and P. Jonas, 2005: How turbulence enhances coalescence of settling particles with applications to rain in clouds. Proc. Roy. Soc. London, 461A, 3059-3088.

Gillespie, D. T., 1972: The stochastic coalescence model for cloud droplet growth. J. Atmos. Sci., 29, 1496-1510.

- 1975: Three models for the coalescence growth of cloud drops. J. Atmos. Sci., 32, 600-607.

Grabowski, W. W., and P. Vaillancourt, 1999: Comments on "Preferential concentration of clouds droplets by turbulence: 
Effects on the early evolution of cumulus cloud droplet spectra." J. Atmos. Sci., 56, 1433-1436.

Grover, S. N., and H. R. Pruppacher, 1985: The effect of vertical turbulent fluctuations in the atmosphere on the collection of aerosol particles by cloud drops. J. Atmos. Sci., 42, 23052318.

Hall, W. D., 1980: A detailed microphysical model within a twodimensional dynamic framework: Model description and preliminary results. J. Atmos. Sci., 37, 2486-2507.

Jameson, A. R., and A. B. Kostinski, 2000: Fluctuation properties of precipitation. Part VI: Observations of hyperfine clustering and drop size distribution structures in three-dimensional rain. J. Atmos. Sci., 57, 373-388.

Johnson, D. B., 1982: The role of giant and ultragiant aerosol particles in warm rain initiation. J. Atmos. Sci., 39, 448-460.

Khain, A., M. Ovtchinnikov, M. Pinsky, A. Pokrovsky, and H. Krugliak, 2000: Notes on the state-of-the-art numerical modeling of cloud microphysics. Atmos. Res., 55, 159-224.

Knight, C. A., J. Vivekanandan, and S. G. Lasher-Trapp, 2002: First radar echoes and the early $Z_{\mathrm{DR}}$ history of Florida cumulus. J. Atmos. Sci., 59, 1454-1472.

Kogan, Y. L., 1993: Drop size separation in numerically simulated convective clouds and its effect on warm rain formation. $J$. Atmos. Sci., 50, 1238-1253.

Kostinski, A. B., and R. A. Shaw, 2001: Scale-dependent droplet clustering in turbulent clouds. J. Fluid Mech., 434, 389-398.

$\longrightarrow$, and - 2005: Fluctuations and luck in droplet growth by coalescence. Bull. Amer. Meteor. Soc., 86, 235-244.

Koziol, A. S., and H. G. Leighton, 1996: The effect of turbulence on the collision rates of small cloud drops. J. Atmos. Sci., 53, 1910-1920.

Kruis, F. E., and K. A. Kusters, 1997: The collision rate of particles in turbulent flow. Chem. Eng. Commun., 58, 201-230.

Langmuir, I., 1948: The production of rain by a chain reaction in cumulus clouds at temperatures above freezing. J. Atmos. Sci., 5, 175-192.

Lasher-Trapp, S. G., W. A. Cooper, and A. M. Blyth, 2005: Broadening of droplet size distributions from entrainment and mixing in a cumulus cloud. Quart. J. Roy. Meteor. Soc., 131, 195-220.

Pinsky, M. B., and A. P. Khain, 1997: Turbulence effects on droplet growth and size distribution in clouds-A review. J. Aerosol Sci., 28, 1177-1214.

$\longrightarrow$, and —, 2002a: Fine structure of cloud droplet concentration as seen from the Fast-FSSP measurements. Part II: Results of in situ observations. J. Appl. Meteor., 42, 65-73.

- , and,$- 2002 \mathrm{~b}$ : Effects of in-cloud nucleation and turbulence on droplet spectrum formation in cumulus clouds. Quart. J. Roy. Meteor. Soc., 128, 501-533.

— and - 2004: Collisions of small drops in a turbulent flow. Part II: Effects of flow accelerations. J. Atmos. Sci., 61, 19261939.

— — - and M. Shapiro, 1999: Collisions of small drops in a turbulent flow. Part I: Collision efficiency. Problem formulation and preliminary results. J. Atmos. Sci., 56, 2585-2600.

,-- , and,- 2000 : Stochastic effects of cloud droplet hydrodynamic interaction in a turbulent flow. Atmos. Res., 53, 131-169.

,-- , B. Grits, and M. Shapiro, 2006: Collisions of small drops in a turbulent flow. Part III: Relative droplet fluxes and swept volumes. J. Atmos. Sci., 63, 2123-2139.

Pruppacher, H. R., and J. D. Klett, 1997: Microphysics of Clouds and Precipitation. 2nd ed. Kluwer Academic, 954 pp.
Reuter, G. W., R. de Villiers, and Y. Yavin, 1988: The collection kernel for two falling cloud drops subjected to random perturbations at a turbulent air flow: A stochastic model. J. Atmos. Sci., 45, 765-773.

Riemer, N., and A. S. Wexler, 2005: Droplets to drops by turbulent coagulation. J. Atmos. Sci., 62, 1962-1975.

—, and - 2006: Reply. J. Atmos. Sci., 63, 2402-2404.

Rogers, R. R., and M. K. Yau, 1989: A Short Course in Cloud Physics. 3rd ed. Butterworth-Heinemann, 290 pp.

Saffman, P. G., and J. S. Turner, 1956: On the collision of drops in turbulent clouds. J. Fluid Mech., 1, 16-30.

Scott, W. T., 1967: Poisson statistics in distributions of coalescing droplets. J. Atmos. Sci., 24, 221-225.

Siebert, H., K. Lehmann, and M. Wendisch, 2006: Observations of small-scale turbulence and energy dissipation rates in the cloudy boundary layer. J. Atmos. Sci., 63, 1451-1466.

Simmel, M., T. Trautmann, and G. Tetzlaff, 2002: Numerical solution of the stochastic collection equation-Comparison of the Linear Discrete Method with other methods. Atmos. Res., 61, 135-148.

Smith, S. A., and P. R. Jonas, 1995: Observations of the turbulent fluxes in fields of cumulus clouds. Quart. J. Roy. Meteor. Soc., 121, 1185-1208.

Srivastava, R. C., 1989: Growth of cloud drops by condensation: A criticism of currently accepted theory and a new approach. $J$. Atmos. Sci., 46, 869-887.

Su, C.-W., S. K. Krueger, P. A. McMurtry, and P. H. Austin, 1998: Linear eddy modeling of droplet spectral evolution during entrainment and mixing in cumulus clouds. Atmos. Res., 4748, 41-58.

Sundaram, S., and L. R. Collins, 1997: Collision statistics in an isotropic particle-laden turbulent suspension. Part 1. Direct numerical simulations. J. Fluid Mech., 335, 75-109.

Szumowski, M. J., R. M. Rauber, H. T. Ochs III, and L. J. Miller, 1997: The microphysical structure and evolution of Hawaiian rainband clouds. Part I: Radar observations of rainbands containing high reflectivity cores. J. Atmos. Sci., 54, 369-385.

Telford, J. W., 1955: A new aspect of coalescence theory. J. Meteor., 12, 436-444.

Vaillancourt, P. A., and M. K. Yau, 2000: Review of particleturbulence interactions and consequences for cloud physics. Bull. Amer. Meteor. Soc., 81, 285-298.

,-- , and W. W. Grabowski, 2001: Microscopic approach to cloud droplet growth by condensation. Part I: Model description and results without turbulence. J. Atmos. Sci., 58, 19451964.

,-- , P. Bartello, and W. W. Grabowski, 2002: Microscopic approach to cloud droplet growth by condensation. Part II: Turbulence, clustering, and condensational growth. J. Atmos. Sci., 59, 3421-3435.

Wang, L.-P., and M. R. Maxey, 1993: Settling velocity and concentration distribution of heavy particles in homogeneous isotropic turbulence. J. Fluid Mech., 256, 27-68.

_ A. S. Wexler, and Y. Zhou, 1998a: On the collision rate of small particles in isotropic turbulence. Part 1. Zero-inertia case. Phys. Fluids, 10, 266-276.

$\longrightarrow, \ldots$, and 1998b: Statistical mechanical descriptions of turbulent coagulation. Phys. Fluids, 10, 2647-2651.

- — - and — 2000: Statistical mechanical descriptions of turbulent coagulation of inertial particles. J. Fluid Mech., 415, $117-153$.

, O. Ayala, S. E. Kasprzak, and W. W. Grabowski, 2005a: Theoretical formulation of collision rate and collision effi- 
ciency of hydrodynamically interacting cloud droplets in turbulent atmosphere. J. Atmos. Sci., 62, 2433-2450.

,-- , and Y. Xue, 2005b: Reconciling the cylindrical formulation with the spherical formulation in the kinematic descriptions of collision kernel. Phys. Fluids, 17, 067 103, doi:10.1063/1.1928647.

, — - - , and W. W. Grabowski, 2006a: Comments on "Droplets to drops by turbulent coagulation." J. Atmos. Sci., 63, 2397-2401.

-, Y. Xue, O. Ayala, and W. W. Grabowski, 2006b: Effects of stochastic coalescence and air turbulence on the size distribution of cloud droplets. Atmos. Res., 82, 416-432.

, C. N. Franklin, O. Ayala, and W. W. Grabowski, 2006c: Probability distributions of angle of approach and relative velocity for colliding droplets in a turbulent flow. J. Atmos. Sci., 63, 881-900.
—, Y. Xue, and W. W. Grabowski, 2007: A bin integral method for solving the kinetic collection equation. J. Comput. Phys., 226, 59-88.

Weil, J. C., R. P. Lawson, and A. R. Rodi, 1993: Relative dispersion of ice crystals in seeded cumuli. J. Appl. Meteor., 32, 1055-1073.

Zaichik, L. I., and V. M. Alipchenkov, 2003: Pair dispersion and preferential concentration of particles in isotropic turbulence. Phys. Fluids, 15, 1776-1787.

_ O. Simonin, and V. M. Alipchenkov, 2003: Two statistical models for predicting collision rates of inertial particles in homogeneous isotropic turbulence. Phys. Fluids, 15, 29953005.

Zhou, Y., A. S. Wexler, and L.-P. Wang, 2001: Modelling turbulent collision of bidisperse inertial particles. J. Fluid Mech., 433, 77-104. 